TRANSACTIONS OF THE

AMERICAN MATHEMATICAL SOCIETY

Volume 355, Number 9 , Pages 3433-3461

S 0002-9947(03)03136-2

Article electronically published on May 29, 2003

\title{
ACCELERATING THE CONVERGENCE OF THE METHOD OF ALTERNATING PROJECTIONS
}

\author{
HEINZ H. BAUSCHKE, FRANK DEUTSCH, HEIN HUNDAL, AND SUNG-HO PARK
}

\begin{abstract}
The powerful von Neumann-Halperin method of alternating projections (MAP) is an algorithm for determining the best approximation to any given point in a Hilbert space from the intersection of a finite number of subspaces. It achieves this by reducing the problem to an iterative scheme which involves only computing best approximations from the individual subspaces which make up the intersection. The main practical drawback of this algorithm, at least for some applications, is that the method is slowly convergent. In this paper, we consider a general class of iterative methods which includes the MAP as a special case. For such methods, we study an "accelerated" version of this algorithm that was considered earlier by Gubin, Polyak, and Raik (1967) and by Gearhart and Koshy (1989). We show that the accelerated algorithm converges faster than the MAP in the case of two subspaces, but is, in general, not faster than the MAP for more than two subspaces! However, for a "symmetric" version of the MAP, the accelerated algorithm always converges faster for any number of subspaces. Our proof seems to require the use of the Spectral Theorem for selfadjoint mappings.
\end{abstract}

\section{INTRODUCTION}

Let $X$ be a (real) Hilbert space, let $M_{1}, M_{2}, \ldots, M_{k}$ be closed (linear) subspaces of $X$ with $M=\bigcap_{1}^{k} M_{i}$, and for any closed subspace $N$ of $X$, let $P_{N}$ denote the orthogonal projection onto $N$. The von Neumann-Halperin method of alternating projections, or MAP for short, is an iterative algorithm for determining the best approximation $P_{M} x$ to $x$ from $M$. It does this by computing the iterates $x_{0}:=x$ and $x_{n}=\left(P_{M_{k}} P_{M_{k-1}} \cdots P_{M_{1}}\right) x_{n-1}=\left(P_{M_{k}} P_{M_{k-1}} \cdots P_{M_{1}}\right)^{n} x$. That is, the iterates $\left(x_{n}\right)$ are obtained by cyclically computing the best approximations onto the individual subspaces $M_{i}(i=1,2, \ldots, k)$. The method is thus most effective when it is "easy" to compute the best approximations from the individual subspaces $M_{i}$. The main theorem governing the MAP is the following.

Theorem (von Neumann [18] for $k=2$, Halperin [15] for $k \geq 2$ ). Let $M_{1}, M_{2}, \ldots$, $M_{k}$ be closed subspaces in the Hilbert space $X$ and let $M:=\bigcap_{1}^{k} M_{i}$. Then

$$
\lim _{n \rightarrow \infty}\left\|\left(P_{M_{k}} P_{M_{k-1}} \cdots P_{M_{1}}\right)^{n} x-P_{M} x\right\|=0 \quad \text { for all } \quad x \in X .
$$

In case $k=2$, this result was rediscovered in at least six other papers (see, e.g., the survey [5]).

Received by the editors July 30, 1999.

2000 Mathematics Subject Classification. Primary 41A65.

Key words and phrases. Alternating projections, cyclic projections, accelerating convergence, best approximation from an intersection of subspaces, Hilbert space. 
Also, as was noted in [5], there are at least ten different areas of mathematics in which the MAP has proved useful. However, the main practical drawback of the MAP appears to be that it is often slowly convergent. Indeed, if $M_{1}+M_{2}$ is not closed, then Franchetti and Light 11] and Bauschke, Borwein, and Lewis [2] have given examples showing that the convergence of $\left(P_{M_{2}} P_{M_{1}}\right)^{n} x$ to $P_{M_{1} \cap M_{2}} x$ can be arbitrarily slow!

Both Gubin, Polyak, and Raik 14 and Gearhart and Koshy 13] have considered a geometrically appealing method to accelerate the MAP, but in neither of these two papers was it proved that the acceleration scheme considered was actually faster than the MAP. In this paper, we will prove that this acceleration scheme is indeed faster than the MAP in the case of two subspaces (i.e., $k=2$ ) (Theorem 3.23). But, perhaps surprisingly, we show that the acceleration scheme may actually be slower than the MAP when $k \geq 3$ (Example 3.24)! In contrast to this, we show that a "symmetric" version of the MAP (i.e., $x_{0}=x$ and $x_{n}=\left(P_{M_{1}} P_{M_{2}} \cdots P_{M_{k}} P_{M_{k-1}} \cdots P_{M_{1}}\right)^{n} x$ for $\left.n=1,2, \ldots\right)$ has an accelerated version which is faster for any $k \geq 2$ (Corollary 3.21).

We should also mention that Dyer [10] and Hanke and Niethammer [16] have considered methods of accelerating the "Kaczmarz method" of solving linear equations. (Recall that Kaczmarz's method may be regarded as the special case of the MAP in the case when $X$ is finite-dimensional and each $M_{i}$ is a hyperplane.)

\section{The Method of iterated PROJECtions}

To provide motivation for the acceleration results to be established later, in this section we give a fairly general convergence result which contains the von NeumannHalperin result as a special case. In the next section, we will consider methods to accelerate this general algorithm.

Unless otherwise stated, the standing assumptions are as follows. Let $X$ be a (real) Hilbert space, $M_{1}, M_{2}, \ldots, M_{k}$ be closed subspaces, $M:=\bigcap_{1}^{k} M_{i}$, and let $P_{i}=P_{M_{i}}$ denote the orthogonal projection onto $M_{i}(i=1,2, \ldots, k)$.

Now let

$$
T:=P_{k} P_{k-1} \cdots P_{1}
$$

denote the composition of the $k$ projections $P_{i}$ taken in increasing order. The well-known von Neumann-Halperin Theorem states that

$$
\lim _{n \rightarrow \infty}\left\|T^{n} x-P_{M} x\right\|=0
$$

for each $x \in X$ (see, more generally, Theorem 2.5 below). Also, it can be shown that

$$
\lim _{n}\left\|\left(T^{*} T\right)^{n} x-P_{M} x\right\|=0
$$

for each $x \in X$ (see Theorem 2.6 below). More generally, suppose $T$ is any bounded linear mapping from $X$ into itself such that

$$
\lim _{n}\left\|T^{n} x-P_{\mathrm{Fix} T} x\right\|=0 \quad \text { for each } x \in X,
$$

where

$$
\operatorname{Fix} T:=\{x \in X \mid T x=x\}
$$

is the fixed point set for $T$.

We will be interested in determining methods to accelerate the convergence of the sequence $\left(T^{n} x\right)$ to $P_{\mathrm{Fix} T} x$. Before we consider such methods, it will provide 
useful motivation to first give some general conditions on the mapping $T$ that will guarantee that (2.0.1) holds.

The mapping $T$ is called nonexpansive if $\|T\| \leq 1$. We first recall that the fixed point sets of $T$ and $T^{*}$ are the same if $T$ is nonexpansive (see Riesz and Sz.-Nagy [19] or Riesz and Sz.-Nagy [20, p. 408]).

Lemma 2.1. Let $T$ be a nonexpansive linear operator on $X$. Then

$$
\operatorname{Fix} T=\operatorname{Fix} T^{*} \text {. }
$$

In fact, $T x=x$ if and only if $\langle T x, x\rangle=\|x\|^{2}$ if and only if $\left\langle x, T^{*} x\right\rangle=\|x\|^{2}$ if and only if $T^{*} x=x$.

Our next observation is a characterization of those linear operators $T$ on $X$ that satisfy (2.0.1). We will use the following notation. If $A$ is any linear operator on $X$, we denote the range and null space of $A$ by $\mathcal{R}(A)$ and $\mathcal{N}(A)$ respectively. We will also use the well-known fact that $\mathcal{N}\left(A^{*}\right)^{\perp}=\overline{\mathcal{R}(A)}$ (see [3] Remarks following Theorem 2.19 on pp. 35-36]).

Theorem 2.2. Let $T$ be a bounded linear operator on $X$, and let $M$ be a closed linear subspace of $X$. Consider the following statements:

(1) $\lim _{n}\left\|T^{n} x-P_{M} x\right\|=0$ for each $x \in X$;

(2) $M=\operatorname{Fix} T$ and $T^{n} x \rightarrow 0$ for each $x \in M^{\perp}$;

(3) $M=\operatorname{Fix} T$ and $T$ is "asymptotically regular", i.e., $T^{n} x-T^{n+1} x \rightarrow 0$ for each $x \in X$.

Then $(1) \Longleftrightarrow(2) \Longrightarrow(3)$. If, in addition, $T$ is nonexpansive, then all three statements are equivalent.

Proof. Suppose (1) holds. If $x \in M$, then $T^{n} x \rightarrow P_{M} x=x$. So by the continuity of $T$,

$$
T x=T\left(\lim T^{n} x\right)=\lim T\left(T^{n} x\right)=\lim T^{n+1} x=P_{M} x=x
$$

implies that $x \in \operatorname{Fix} T$, i.e., $M \subset \operatorname{Fix} T$.

Conversely, let $y \in \operatorname{Fix} T$. Then $T y=y$ and an easy induction shows that $y=T^{n} y$ for each $n$. Thus $y=T^{n} y \rightarrow P_{M} y$ which implies $y=P_{M} y \in M$. That is, Fix $T \subset M$. Hence $M=\operatorname{Fix} T$.

If $x \in M^{\perp}$, then

$$
T^{n} x=T^{n}\left(P_{M \perp} x\right) \rightarrow P_{M}\left(P_{M \perp} x\right)=0 .
$$

This proves $(2)$.

Now assume (2) holds and let $x \in X$. Then

$$
T^{n} x=T^{n}\left(P_{M} x+P_{M \perp} x\right)=T^{n}\left(P_{M} x\right)+T^{n}\left(P_{M \perp} x\right)=P_{M} x+T^{n}\left(P_{M \perp}\right) \rightarrow P_{M} x .
$$

Thus (1) holds, and this establishes the equivalence of (1) and (2).

Now suppose that (2) holds and $x \in X$. By the equivalence of (1) and (2), we have that $T^{n} x \rightarrow P_{M} x$ and so $T^{n} x-T^{n+1} x \rightarrow P_{M} x-P_{M} x=0$. Thus $T$ is asymptotically regular, and hence (3) holds.

This proves the first statement of the theorem. To complete the proof, suppose (3) holds and let $T$ be nonexpansive. Then Fix $T^{*}=\operatorname{Fix} T=M$ by Lemma 2.1. Then for any $x \in X$, we have that $T^{n}(x-T x)=T^{n} x-T^{n+1} x \rightarrow 0$. Hence $T^{n} y \rightarrow 0$ for every $y \in \mathcal{R}(I-T)$ which implies, since $\left\|T^{n}\right\| \leq 1$ by nonexpansiveness, that $T^{n} y \rightarrow 0$ for every

$$
y \in \overline{\mathcal{R}(I-T)}=\mathcal{N}\left(I-T^{*}\right)^{\perp}=\left(\operatorname{Fix} T^{*}\right)^{\perp}=M^{\perp} .
$$


Thus, for any $x \in X$,

$T^{n} x=T^{n}\left(P_{M} x+P_{M^{\perp}} x\right)=T^{n}\left(P_{M} x\right)+T^{n}\left(P_{M^{\perp}} x\right)=P_{M} x+T^{n}\left(P_{M^{\perp}} x\right) \rightarrow P_{M} x$,

and this proves that (1) holds.

Remark. Statement (3) does not imply statement (1) in general. To see this, let $X$ denote the Euclidean plane and let $e_{1}=(1,0)$ and $e_{2}=(0,1)$ denote the canonical orthonormal basis vectors in $X$. Defining $T: X \rightarrow X$ by $T x=[x(1)+x(2)] e_{1}$, it is easy to verify that $T^{n} x=T x$ for every $n \in \mathbb{N}$ and every $x \in X$, so that $T$ is asymptotically regular, $M:=$ Fix $T=\operatorname{span} e_{1}, T^{n}\left(e_{1}+e_{2}\right)=2 e_{1}$ for every $n$, but $P_{M}\left(e_{1}+e_{2}\right)=e_{1} \neq 2 e_{1}=T^{n}\left(e_{1}+e_{2}\right)$ for every $n$. Thus, $T^{n} x \nrightarrow P_{M}(x)$ when $x=e_{1}+e_{2}$.

Corollary 2.3. Let $T$ be nonexpansive on $X$ and $M=$ Fix $T$. Then

$$
\lim _{n \rightarrow \infty}\left\|T^{n} x-P_{M} x\right\|=0 \quad \text { for all } \quad x \in X
$$

if and only if $T$ is asymptotically regular.

Lemma 2.4. Let $M_{1}, M_{2}, \ldots, M_{k}$ be closed subspaces of the Hilbert space $X$, let $M:=\bigcap_{1}^{k} M_{i}$ and let $T:=P_{M_{k}} P_{M_{k-1}} \cdots P_{M_{1}}$. Then $T$ is nonexpansive and

$$
\operatorname{Fix} T=\operatorname{Fix} T^{*}=\operatorname{Fix}\left(T T^{*}\right)=\operatorname{Fix}\left(T^{*} T\right)=M .
$$

Proof. For simplicity, let $P_{i}=P_{M_{i}}$. Since $T$ is the product of nonexpansive operators, $T$ is nonexpansive. If $x \in M$, then $x \in M_{i}$ for each $i$ so that $P_{i} x=x$ for each $i$ and hence $T x=x$. That is, $M \subset \operatorname{Fix} T$. Conversely, if $z \in \operatorname{Fix} T$, then $T z=z$. Thus, $P_{k} P_{k-1} \cdots P_{1} z=z$. We have $P_{i} z=z$ if and only if $\left\|P_{i} z\right\|=\|z\|$ (using the fact that $\left.\|z\|^{2}=\left\|P_{i} z\right\|^{2}+\left\|z-P_{i} z\right\|^{2}\right)$. If $z \notin M$, let $i$ be the smallest index such that $z \notin M_{i}$. Then $P_{i} z \neq z ;$ so $\left\|P_{i} z\right\|<\|z\|$ and $z=P_{k} \cdots P_{i} P_{i-1} \cdots P_{1} z=P_{k} \cdots P_{i} z$ implies that $\|z\|=\left\|P_{k} \cdots P_{i} z\right\| \leq\left\|P_{i} z\right\|<\|z\|$, which is absurd. Thus, $z \in M$. This proves that $M=$ Fix $T$. By Lemma $2.1, M=$ Fix $T^{*}$.

Since $T^{*}=P_{1} P_{2} \cdots P_{k}$, we see that $T T^{*}=P_{k} P_{k-1} \cdots P_{1} P_{2} \cdots P_{k}$ and $T^{*} T=$ $P_{1} P_{2} \cdots P_{k} P_{k-1} \cdots P_{1}$, and the same proof as above shows that Fix $T T^{*}=M=$ Fix $T^{*} T$.

A useful sufficient condition that guarantees that (2.0.1) holds is essentially contained in Halperin [15]. It also is explicit in Smarzewski [21] and can be stated as follows. (We include a brief proof since, as far as we know, the paper 21 has not been published.) Recall that $T: X \rightarrow X$ is called nonnegative if $\langle T x, x\rangle \geq 0$ for all $x \in X$.

Theorem 2.5. Let $T_{1}, T_{2}, \ldots, T_{k}$ be selfadjoint, nonnegative, and nonexpansive bounded linear operators on the Hilbert space $X$. Let $T:=T_{1} T_{2} \cdots T_{k}$ and $M=$ Fix $T$. Then Fix $T=\bigcap_{1}^{k} \operatorname{Fix} T_{i}$ and

$$
\lim _{n}\left\|T^{n} x-P_{M} x\right\|=0 \quad \text { for every } \quad x \in X .
$$

Proof. Since $T$ is nonexpansive, Corollary 2.3 implies that it suffices to show that $T$ is asymptotically regular. Toward this end, note that for each $i, I-T_{i}$ is nonnegative (and selfadjoint) since

$$
\left\langle\left(I-T_{i}\right) x, x\right\rangle=\left\langle x-T_{i} x, x\right\rangle=\|x\|^{2}-\left\langle T_{i} x, x\right\rangle \geq\|x\|^{2}-\left\|T_{i}\right\|\|x\|^{2} \geq 0 .
$$


It follows from a result of Riesz (see [4, Theorem 4.6.4, p. 163]) that $T_{i}\left(I-T_{i}\right)$ is also nonnegative. Hence,

$$
\begin{aligned}
\|x\|^{2} & =\left\|x-T_{i} x+T_{i} x\right\|^{2}=\left\|x-T_{i} x\right\|^{2}+2\left\langle x-T_{i} x, T_{i} x\right\rangle+\left\|T_{i} x\right\|^{2} \\
& =\left\|x-T_{i} x\right\|^{2}+2\left\langle T_{i}\left(I-T_{i}\right) x, x\right\rangle+\left\|T_{i} x\right\|^{2} \geq\left\|x-T_{i} x\right\|^{2}+\left\|T_{i} x\right\|^{2} .
\end{aligned}
$$

Thus, for each $x \in X$,

$$
\left\|x-T_{i} x\right\|^{2} \leq\|x\|^{2}-\left\|T_{i} x\right\|^{2} \text { for each } i .
$$

By repeated application of (2.5.2), we deduce that

$$
\begin{aligned}
\|x\|^{2}- & \|T x\|^{2} \\
& =\|x\|^{2}-\left\|T_{k} x\right\|^{2}+\left\|T_{k} x\right\|^{2}-\left\|T_{k-1} T_{k} x\right\|^{2}+\cdots+\left\|T_{2} \cdots T_{k} x\right\|^{2}-\|T x\|^{2} \\
& \geq\left\|x-T_{k} x\right\|^{2}+\left\|T_{k} x-T_{k-1} T_{k} x\right\|^{2}+\cdots+\left\|T_{2} \cdots T_{k} x-T x\right\|^{2} \\
& =k\left[\frac{1}{k}\left\|x-T_{k} x\right\|^{2}+\frac{1}{k}\left\|T_{k} x-T_{k-1} T_{k} x\right\|^{2}+\cdots+\frac{1}{k}\left\|T_{2} \cdots T_{k} x-T x\right\|^{2}\right] \\
& \geq k\left\|\frac{1}{k}\left(x-T_{k} x+T_{k} x-T_{k-1} T_{k} x+\cdots+T_{2} \cdots T_{k} x-T x\right)\right\|^{2}
\end{aligned}
$$

(by convexity of $\|\cdot\|^{2}$ )

$=\frac{1}{k}\|x-T x\|^{2}$.

That is,

$$
\|x-T x\|^{2} \leq k\left(\|x\|^{2}-\|T x\|^{2}\right) \text { for every } x \in X .
$$

Since $T$ is nonexpansive, we see that the sequence $\left(\left\|T^{n} x\right\|\right)_{n=1}^{\infty}$ is nonincreasing for every $x \in X$ and so it must converge: $\left\|T^{n} x\right\| \rightarrow \rho \geq 0$. Now apply (2.5.3) with $x$ replaced by $T^{n} x$ to obtain that

$$
\left\|T^{n} x-T^{n+1} x\right\|^{2} \leq k\left(\left\|T^{n} x\right\|^{2}-\left\|T^{n+1} x\right\|^{2}\right) \rightarrow 0 \quad \text { as } n \rightarrow \infty .
$$

This proves that $T$ is asymptotically regular.

Lemma 2.4 and Theorem 2.5 immediately imply the following two results. The first is the "von Neumann-Halperin theorem" stated in the Introduction, while the second shows that a symmetric version of the MAP also converges.

Theorem 2.6. Let $M_{1}, M_{2}, \ldots, M_{k}$ be closed subspaces of the Hilbert space $X$, and let $M=\bigcap_{1}^{k} M_{i}$. Then, for each $x \in X$,

$$
\lim _{n}\left\|\left(P_{M_{k}} P_{M_{k-1}} \cdots P_{M_{1}}\right)^{n} x-P_{M} x\right\|=0 .
$$

Theorem 2.7. Let $M_{1}, M_{2}, \ldots, M_{k}$ be closed subspaces of the Hilbert space $X$, and let $M=\bigcap_{1}^{k} M_{i}$. Then, for each $x \in X$,

$$
\lim _{n}\left\|\left(P_{M_{1}} P_{M_{2}} \cdots P_{M_{k}} P_{M_{k-1}} \cdots P_{M_{1}}\right)^{n} x-P_{M} x\right\|=0 .
$$

Using Theorems 2.6 and 2.7, we see that two important examples of operators $T$ which satisfy $(2.0 .1)$ are $T=Q$ and $T=Q^{*} Q$, where $Q:=P_{M_{k}} P_{M_{k-1}} \cdots P_{M_{1}}$. 


\section{AcCeleration methods}

Throughout this section, unless explicitly stated otherwise, we assume that $T$ is a nonexpansive linear operator on $X$ and $M:=\operatorname{Fix} T$. Hence, Fix $T^{*}=M$ also (by Lemma 2.1). Moreover, $M_{i}$ will always denote a closed linear subspace of $X$ and $P_{i}=P_{M_{i}}$.

In this section, we develop our main results concerned with accelerating the method given by (2.0.1). That is, if $T$ is an operator such that (2.0.1) holds (or equivalently, that $T$ is asymptotically regular), how can we modify the iterates suggested by this algorithm so as to converge faster to $P_{M} x$ ?

Definition 3.1. The accelerated mapping $A_{T}$ of $T$ is defined on $X$ by

$$
A_{T}(x):=t_{x} T x+\left(1-t_{x}\right) x
$$

where

$$
t_{x}=t_{x, T}:= \begin{cases}\frac{\langle x, x-T x\rangle}{\|x-T x\|^{2}} & \text { if } T x \neq x \\ 1 & \text { if } T x=x .\end{cases}
$$

We will consider two classes of iterative algorithms to compute $P_{M}(x)$ for any given $x \in X$. They are described as follows. The standard or "unaccelerated" algorithm: $x_{0}=x$ and

$$
x_{n}=T\left(x_{n-1}\right)=T^{n}(x) \quad(n=1,2, \ldots),
$$

and its "accelerated" counterpart: $x_{0}=x, \quad x_{1}=T\left(x_{0}\right)$, and

$$
x_{n}=A_{T}\left(x_{n-1}\right)=A_{T}^{n-1}(T x) \quad(n=1,2, \ldots) .
$$

In particular, we will give a detailed analysis of these algorithms when $T=P_{k} P_{k-1}$ $\cdots P_{1}$ and when $T=\left(P_{k} P_{k-1} \cdots P_{1}\right)^{*}\left(P_{k} P_{k-1} \cdots P_{1}\right)$. This acceleration scheme was suggested by Gubin et al [14] and Gearhart and Koshy 13] in the particular case when $T$ is a product of projections. The motivation for using the mapping $A_{T}$ is that $A_{T}(x)$ is that point on the line through the points $x$ and $T x$ which is closest to $P_{M} x$ (see Theorem 3.7 below).

A remark is in order as to why, in the accelerated algorithm, we first apply $T$ to $x_{0}$ rather than first applying $A_{T}$. That is, why didn't we define the accelerated algorithm by $x_{n}=A_{T}^{n}\left(x_{0}\right)$ for $n \geq 0$ rather than $x_{n+1}=A_{T}^{n}\left(T x_{0}\right)$ for $n \geq 0$ ? The simple answer is that, besides being the one suggested in [14] and [13, the one we defined performs better. Indeed, it is not hard to see that if $T$ is the product of two orthogonal projections onto two 1-dimensional (nonorthogonal) subspaces in the Euclidean plane, then the accelerated algorithm converges in two steps, that is, $A_{T}(T x)=P_{M} x$ for any starting point $x$. However, for any choice of $x$ which is not in the range of $T$, none of the terms of the sequence $\left(A_{T}^{n}(x)\right)$ is equal to $P_{M} x$. That is, the sequence $x_{n}=A_{T}^{n}(x)$ does not converge to $P_{M} x$ in a finite number of steps.

Definition 3.2. The classical von Neumann-Halperin method of alternating projections, or MAP for short, corresponds to (3.1.3) in the case when $T=$ $P_{k} P_{k-1} \cdots P_{1}$.

The accelerated method of alternating projections, or the accelerated MAP for short, is the algorithm (3.1.4) in the case when $T=P_{k} P_{k-1} \cdots P_{1}$.

The symmetric method of alternating projections, or symmetric MAP for short, is just (3.1.3) in the case when $T=\left(P_{k} P_{k-1} \cdots P_{1}\right)^{*}\left(P_{k} P_{k-1} \cdots P_{1}\right)$. 
The accelerated symmetric method of alternating projections, or accelerated symmetric MAP for short, is the algorithm (3.1.4) in the case when $T=\left(P_{k} P_{k-1} \cdots P_{1}\right)^{*}\left(P_{k} P_{k-1} \cdots P_{1}\right)$.

Lemma 3.3. Let $x \in X$. Then

(1) $t A_{T}(x)+(1-t) x-x \in M^{\perp} \cap\left(A_{T}(x)\right)^{\perp}$ for every $t \in \mathbb{R}$.

(2) $T x-x \in M^{\perp} \cap\left(A_{T}(x)\right)^{\perp}$.

(3) $A_{T}(x)-x \in M^{\perp} \cap\left(A_{T}(x)\right)^{\perp}$.

(4) $T\left(M^{\perp}\right) \subset M^{\perp}$ and $A_{T}\left(M^{\perp}\right) \subset M^{\perp}$.

(5) $A_{T}(x)-T x \in M^{\perp} \cap\left(A_{T}(x)\right)^{\perp}$.

(6) $T x-P_{M} x \in M^{\perp}$.

Proof. (1) Since $t A_{T}(x)+(1-t) x-x=t t_{x}(T x-x)$, it suffices to verify (2).

(2) If $T x=x$, then (2) is trivial. Thus we may assume that $T x \neq x$. Let $y \in M$. Then since $T y=y=T^{*} y$, we have

$$
\langle T x-x, y\rangle=\left\langle x, T^{*} y\right\rangle-\langle x, y\rangle=\langle x, y\rangle-\langle x, y\rangle=0 .
$$

Thus $T x-x \in M^{\perp}$. Also,

$$
\begin{aligned}
\left\langle T x-x, A_{T}(x)\right\rangle & =\left\langle T x-x, t_{x} T x+\left(1-t_{x}\right) x\right\rangle=t_{x}\langle T x-x, T x-x\rangle+\langle T x-x, x\rangle \\
& =-\frac{\langle x, T x-x\rangle}{\|x-T x\|^{2}}\|T x-x\|^{2}+\langle T x-x, x\rangle=0 ;
\end{aligned}
$$

so $T x-x \in\left(A_{T}(x)\right)^{\perp}$.

(3) Take $t=1$ in (1).

(4) This follows from (2) and (3).

(5) Since $A_{T}(x)-T x=\left(t_{x}-1\right)(T x-x)$, the result follows from (2).

(6) Using part (2), we get

$$
T x-P_{M} x=(T x-x)+\left(x-P_{M} x\right) \in M^{\perp}+M^{\perp}=M^{\perp} .
$$

Lemma 3.4. For every $x \in X$ and $n \in \mathbb{N}:=\{1,2, \ldots$,$\} ,$

(1) $P_{M}\left(A_{T}(x)\right)=P_{M} x$;

(2) $P_{M}(T x)=P_{M} x$;

(3) $P_{M}\left(A_{T}^{n-1}(T x)\right)=P_{M} x$;

(4) $P_{M}\left(T^{n} x\right)=P_{M} x$.

Proof. We use the well-known fact that $P_{M}\left(M^{\perp}\right)=\{0\}$. Since $T x-x \in M^{\perp}$ and $A_{T}(x)-x \in M^{\perp}$ by Lemma 3.3, it follows that $0=P_{M}(T x-x)=P_{M}(T x)-P_{M} x$ and $0=P_{M}\left(A_{T}(x)-x\right)=P_{M}\left(A_{T}(x)\right)-P_{M} x$. Hence (1) and (2) follow.

(3) and (4) follow by a repeated application of (1) and (2).

Lemma 3.5. For each $x \in X$ and $y \in M$,

$$
\left\|A_{T}(x)-y\right\|^{2}=\|x-y\|^{2}-\left\|x-A_{T}(x)\right\|^{2} .
$$

In particular,

$$
\operatorname{Fix} A_{T}=\left\{x \in X \mid\left\|A_{T}(x)\right\|=\|x\|\right\}
$$

and

$$
\left\|A_{T}(x)\right\|^{2}= \begin{cases}\|x\|^{2} & \text { if } x \in M, \\ \|x\|^{2}-\frac{\langle x, x-T x\rangle^{2}}{\|x-T x\|^{2}} & \text { if } x \notin M .\end{cases}
$$


Proof. Using Lemma 3.3, we deduce that

$$
\|x-y\|^{2}=\left\|\left(x-A_{T}(x)\right)+\left(A_{T}(x)-y\right)\right\|^{2}=\left\|x-A_{T}(x)\right\|^{2}+\left\|A_{T}(x)-y\right\|^{2} ;
$$

so (3.5.1) holds. Take $y=0$ in (3.5.1) to obtain (3.5.2). Finally, take $y=0$ in (3.5.1) and note that $\left\|x-A_{T}(x)\right\|^{2}=\frac{\langle x, x-T x\rangle^{2}}{\|x-T x\|^{2}}$ if $x \notin M$ and $\left\|x-A_{T}(x)\right\|^{2}=0$ if $x \in M$. This yields (3.5.3).

Lemma 3.6. The following statements are equivalent:

(1) $T x \in M$;

(2) $T x=P_{M} x$

(3) $T^{n} x \in M$ for every $n \geq 1$.

Proof. (1) $\Longrightarrow(2)$. If $T x \in M$, then $T x=P_{M}(T x)=P_{M} x$ using Lemma 3.4(2).

(2) $\Longrightarrow$ (3). If $T x=P_{M} x$, then $T x \in M$. Thus, (3) holds when $n=1$. We proceed by induction. If $T^{n} x \in M$ for some $n \geq 1$, then since $M=\operatorname{Fix} T$, we have that

$$
T^{n+1} x=T\left(T^{n} x\right)=T^{n} x \in M .
$$

This completes the induction.

$(3) \Longrightarrow(1)$. Take $n=1$.

The affine hull of a nonempty set $S$, denoted by aff $(S)$, is the intersection of the collection of all affine sets which contain $S$. (Recall that an affine set is any translation of a subspace.) Equivalently, $\operatorname{aff}(S)=\{\alpha x+(1-\alpha) y \mid x, y \in S, \alpha \in \mathbb{R}\}$.

Theorem 3.7. For each $x \in X$ and $y \in M$, we have

$$
\begin{gathered}
\left\|A_{T}(x)-y\right\|^{2}=\|t T x+(1-t) x-y\|^{2}-\left(t-t_{x}\right)^{2}\|T x-x\|^{2} \quad \text { for each } t \in \mathbb{R}, \\
\left\|A_{T}(x)-y\right\|=\min _{t \in \mathbb{R}}\|t T x+(1-t) x-y\|,
\end{gathered}
$$

and the minimum is attained precisely when either $t=t_{x}$ if $x \notin M$ or at every $t \in \mathbb{R}$ if $x \in M$. Moreover,

$$
d\left(A_{T}(x), M\right)=\min _{t \in \mathbb{R}} d(t T x+(1-t) x, M) ;
$$

in other words, $A_{T}(x)$ is the unique point in aff $\{x, T x\}$ which is closest to $M$.

$$
\left\|A_{T}(x)\right\|=\min _{t \in \mathbb{R}}\|t T x+(1-t) x\|
$$

in other words, $A_{T}(x)$ is the unique point in aff $\{x, T x\}$ having minimal norm. In particular,

$$
\left\|A_{T}(x)\right\| \leq \min \{\|x\|,\|T x\|\} .
$$

Proof. Using Lemma 3.3, we can write

$$
\begin{aligned}
\|t T x+(1-t) x-y\|^{2} & =\left\|t T x+(1-t) x-A_{T}(x)+A_{T}(x)-y\right\|^{2} \\
& =\left\|\left(t-t_{x}\right)(T x-x)+\left(A_{T}(x)-y\right)\right\|^{2} \\
& =\left(t-t_{x}\right)^{2}\|T x-x\|^{2}+\left\|A_{T}(x)-y\right\|^{2},
\end{aligned}
$$

which proves (3.7.1). Equation (3.7.2) follows immediately from (3.7.1). Moreover, (3.7.3) follows by taking the infimum over all $y \in M$ in (3.7.2). Finally, (3.7.4) follows from (3.7.2) by taking $y=0$. 
While $A_{T}$ is not linear in general, it does share some important properties of the linear mapping $P_{M}$. Namely, it is continuous, homogeneous, and "additive modulo $M "$. These are recorded in parts (5), (4), and (3), respectively, of the following lemma.

Lemma 3.8. Let $x \in X$ and $y \in M$. Then:

(1) $t_{x+y}=t_{x}$.

(2) $t_{\alpha x}=t_{x}$ for every $\alpha \neq 0$.

(3) $A_{T}^{n}(x+y)=A_{T}^{n}(x)+y$ for every $n \in \mathbb{N}$. In particular, $A_{T}(x+y)=A_{T}(x)+y$ and $A_{T}(y)=y$.

(4) $A_{T}(\alpha x)=\alpha A_{T}(x)$ for every $\alpha \in \mathbb{R}$.

(5) $A_{T}$ is continuous.

Proof. (1) If $x \in M$, then $x+y \in M$ and $t_{x+y}=1=t_{x}$. If $x \notin M$, then $x+y \notin M$, and so,

$$
t_{x+y}=\frac{\langle x+y, x+y-T(x+y)\rangle}{\|x+y-T(x+y)\|^{2}}=\frac{\langle x+y, x-T x\rangle}{\|x-T x\|^{2}}=\frac{\langle x, x-T x\rangle}{\|x-T x\|^{2}}=t_{x}
$$

using Lemma 3.3(2).

(2) Let $\alpha \neq 0$. If $x \in M$, then $\alpha x \in M$ and $t_{\alpha x}=1=t_{x}$. If $x \notin M$, then $\alpha x \notin M$ and

$$
t_{\alpha x}=\frac{\langle\alpha x, \alpha x-T(\alpha x)\rangle}{\|\alpha x-T(\alpha x)\|^{2}}=\frac{\langle x, x-T x\rangle}{\|x-T x\|^{2}}=t_{x} .
$$

(3) When $n=1$,

$$
\begin{aligned}
A_{T}(x+y) & =t_{x+y} T(x+y)+\left(1-t_{x+y}\right)(x+y) \\
& =t_{x}(T x+T y)+\left(1-t_{x}\right)(x+y) \text { using part }(1) \\
& =t_{x} T x+\left(1-t_{x}\right) x+t_{x} y+\left(1-t_{x}\right) y \\
& =A_{T}(x)+y
\end{aligned}
$$

Now assume (3) holds for some $n \geq 1$. Then

$$
\begin{aligned}
A_{T}^{n+1}(x+y) & =A_{T}\left[A_{T}^{n}(x+y)\right]=A_{T}\left[A_{T}^{n}(x)+y\right] \\
& =A_{T}\left(A_{T}^{n}(x)\right)+y \quad \text { by the case } n=1 \\
& =A_{T}^{n+1}(x)+y
\end{aligned}
$$

so the result holds for $n+1$.

(4) If $\alpha \neq 0$, then by (2),

$$
A_{T}(\alpha x)=t_{\alpha x} T(\alpha x)+\left(1-t_{\alpha x}\right)(\alpha x)=t_{x}[\alpha T(x)]+\left(1-t_{x}\right)[\alpha x]=\alpha A_{T}(x) .
$$

Since $A_{T}(0)=0$, the result also holds when $\alpha=0$.

(5) If $x \in X \backslash M$ and $x_{n} \rightarrow x$, then since $X \backslash M$ is open, $x_{n} \notin M$ eventually, and so,

$$
t_{x_{n}}=\frac{\left\langle x_{n}, x_{n}-T x_{n}\right\rangle}{\left\|x_{n}-T x_{n}\right\|^{2}} \rightarrow \frac{\langle x, x-T x\rangle}{\|x-T x\|^{2}}=t_{x}
$$


and hence $A_{T}$ is continuous at $x$. If $x \in M$ and $\epsilon>0$, let $y \in X$ with $\|y-x\|<\epsilon / 3$. Then $\left\|P_{M} x-P_{M} y\right\| \leq\|x-y\|<\epsilon / 3$ and

$$
\begin{aligned}
\left\|A_{T}(x)-A_{T}(y)\right\| & =\left\|x-A_{T}(y)\right\| \leq\left\|x-P_{M} y\right\|+\left\|P_{M} y-A_{T}(y)\right\| \\
& =\left\|x-P_{M} y\right\|+\left\|A_{T}\left(y-P_{M} y\right)\right\| \quad \text { by part }(3) \\
& \leq\left\|x-P_{M} y\right\|+\left\|y-P_{M} y\right\| \quad \text { by }(3.7 .5) \\
& =\left\|P_{M} x-P_{M} y\right\|+\left\|y-P_{M} y\right\| \\
& <\frac{\epsilon}{3}+\|y-x\|+\left\|x-P_{M} y\right\| \\
& <\frac{2 \epsilon}{3}+\left\|P_{M} x-P_{M} y\right\|<\epsilon .
\end{aligned}
$$

This proves that $A_{T}$ is continuous at $x$.

Remark. We note that, while $A_{T}$ is continuous, it is not uniformly continuous, in general, unlike a linear operator. For example, let $X=\ell_{2}$, let $\left\{e_{n} \mid n=0,1,2, \ldots\right\}$ be an orthonormal basis for $X$, and define $T$ on $X$ by $T x=\sum_{0}^{\infty}\left\langle x, e_{n}\right\rangle n /(n+1) e_{n}$. Setting $x_{n}=(1 / n) e_{0}+((n+1) / n) e_{n}$ and $y_{n}=e_{n}$ for all $n \geq 1$, we get that $\left\|x_{n}-y_{n}\right\|=(\sqrt{2} / n) \rightarrow 0$. But using the readily deduced facts that $A_{T}\left(y_{n}\right)=0$ and $A_{T}\left(x_{n}\right)=(1 / 2)\left(e_{n}-e_{0}\right)$ for all $n$, we obtain that $\left\|A_{T}\left(x_{n}\right)-A_{T}\left(y_{n}\right)\right\|=(\sqrt{2} / 2)$ for all $n \geq 1$.

Lemma 3.9. (1) $t_{x} \geq \frac{1}{2}$ for all $x \in X$; and

(2) $\operatorname{Fix} A_{T}=M(=\operatorname{Fix} T)$.

Proof. (1) If $x \in M$, then $t_{x}=1$. If $x \notin M$, then the quadratic function,

$$
q(t):=\|t(T x-x)+x\|^{2}=a t^{2}+b t+c,
$$

where $a:=\|T x-x\|^{2}>0, b:=2\langle x, T x-x\rangle$, and $c:=\|x\|^{2}$ is strictly convex and attains its minimum at the unique point $t$ when $q^{\prime}(t)=0$; that is, when $t=t_{\min }:=-\frac{b}{2 a}$. Hence,

$$
t_{\min }=-\frac{2\langle x, T x-x\rangle}{2\|T x-x\|^{2}}=\frac{\langle x, x-T x\rangle}{\|x-T x\|^{2}}=: t_{x} .
$$

But $c=q(0)=\|x\|^{2}$ and $\|T x\|^{2}=q(1)=a+b+c=a+b+\|x\|^{2}$ implies that $-b=a+\|x\|^{2}-\|T x\|^{2}$ and hence

$$
t_{x}=t_{\min }=\frac{-b}{2 a}=\frac{a+\|x\|^{2}-\|T x\|^{2}}{2 a}=\frac{1}{2}+\frac{\|x\|^{2}-\|T x\|^{2}}{2 a} \geq \frac{1}{2} .
$$

(2) $x \in \operatorname{Fix} A_{T}$ if and only if $x=t_{x} T x+\left(1-t_{x}\right) x$ if and only if $t_{x}(T x-x)=0$ if and only if $T x-x=0$ (using part (1)) if and only if $x \in \operatorname{Fix} T=M$.

Remarks. The lower bound $\frac{1}{2}$ for $t_{x}$ is sharp. To see this, take $T=-I$ and note that $t_{x}=\frac{1}{2}$ for every $x \in X \backslash\{0\}$. Also, if we relax the condition that $T$ be nonexpansive and consider $T=\lambda I$ for $\lambda \neq 1$, we deduce that $t_{x, \lambda I}=\frac{1}{1-\lambda}$ for each $x \neq 0$. By varying $\lambda$, we see that $t_{x, \lambda I}$ can take on every nonzero value.

Definition 3.10. Define $f=f_{T}: X \rightarrow \mathbb{R}^{+}:=\{\alpha \in \mathbb{R} \mid \alpha \geq 0\}$ by

$$
f(x):= \begin{cases}\frac{\left\|A_{T}(x)-P_{M} x\right\|}{\left\|T x-P_{M} x\right\|} & \text { if } T x \notin M, \\ 0 & \text { if } T x \in M .\end{cases}
$$


Lemma 3.11. For each $x \in X$, we have $0 \leq f(x) \leq 1$ and

$$
\left\|A_{T}(x)-P_{M} x\right\|=f(x)\left\|T x-P_{M} x\right\| .
$$

Proof. This is immediate from (3.7.2) with $y=P_{M} x$.

Lemma 3.12. $T$ commutes with $P_{M}$ and $P_{M^{\perp}}$.

Proof. For each $x \in X$,

$$
\begin{aligned}
P_{M} T x & =P_{M} T\left(P_{M} x+P_{M^{\perp}} x\right)=P_{M}\left[T\left(P_{M} x\right)+T\left(P_{M^{\perp}} x\right)\right] \\
& =P_{M}^{2} x \quad \text { since } T\left(M^{\perp}\right) \subset M^{\perp} \text { by Lemma } 3.3(4) \\
& =P_{M} x=T P_{M} x .
\end{aligned}
$$

Thus, $T$ commutes with $P_{M}$ and, since $P_{M^{\perp}}=I-P_{M}$, it follows that $T$ also commutes with $P_{M \perp}$.

Definition 3.13. Let $T$ be a nonexpansive linear operator on $X, M=$ Fix $T$, and for any $n \in \mathbb{N}$, let $c_{n}(T)$ denote the norm of the linear operator $\left(T P_{M^{\perp}}\right)^{n}$ :

$$
c_{n}(T):=\left\|\left(T P_{M^{\perp}}\right)^{n}\right\| .
$$

We will often write $c(T)$ instead of $c_{1}(T)$. Note that if $T=P_{M_{k}} P_{M_{k-1}} \cdots P_{M_{1}}$, then $M:=\bigcap_{1}^{k} M_{i}=$ Fix $T$ and

$$
c(T)=\left\|P_{M_{k}} P_{M_{k-1}} \cdots P_{M_{1}} P_{M^{\perp}}\right\|=: c\left(M_{1}, M_{2}, \ldots M_{k}\right)
$$

is just the cosine of the angle of the $k$-tuple $\left(M_{1}, M_{2}, \ldots, M_{k}\right)$ defined by Bauschke, Borwein, and Lewis [2]. It was established in 2] that $c(T)<1$ if and only if $M_{1}^{\perp}+M_{2}^{\perp}+\cdots+M_{k}^{\perp}$ is closed. When $k=2$,

(3.13.3) $\quad c\left(P_{M_{2}} P_{M_{1}}\right)=\left\|P_{M_{2}} P_{M_{1}} P_{M^{\perp}}\right\|=c\left(M_{1}, M_{2}\right)=c\left(M_{2}, M_{1}\right)=c\left(P_{M_{1}} P_{M_{2}}\right)$

is just the ordinary cosine of the angle between the subspaces $M_{1}$ and $M_{2}$ (see [12] or [7]).

Lemma 3.14. Let $T$ be nonexpansive on $X$ and $M=\operatorname{Fix} T$. Then

(1) $c_{n}(T)=\left\|T^{n}-P_{M}\right\|$ for every $n \in \mathbb{N}$. In particular,

(3.14.1) $\left\|T^{n} x-P_{M} x\right\| \leq c_{n}(T)\left\|x-P_{M} x\right\| \quad$ for every $n \in \mathbb{N}$, and $x \in X$,

and $c_{n}(T)$ is the smallest constant independent of $x$ for which (3.14.1) is valid.

(2) $\left\|T^{n} y\right\| \leq c_{n}(T)\|y\|$ for every $y \in M^{\perp}$;

(3) $c_{n}(T) \leq c(T)^{n}$ for every $n$;

(4) $c\left(T^{*} T\right) \leq c(T)^{2}$ and $c\left(T^{*} T\right)=c(T)^{2}$ if $\operatorname{Fix}\left(T^{*} T\right)=\operatorname{Fix} T$. In particular, if $T=P_{M_{k}} P_{M_{k-1}} \cdots P_{M_{1}}$, then

$$
c\left(T^{*} T\right)=c(T)^{2}
$$

(5) $\left\|A_{T}(x)-P_{M} x\right\| \leq f(x) c(T)\left\|x-P_{M} x\right\|$ for every $x \in X$.

Proof. (1) By Lemma 3.12, $T$ commutes with $P_{M}$ and $T P_{M}=P_{M}=P_{M} T$. Thus,

$$
c_{n}(T)=\left\|\left(T P_{M^{\perp}}\right)^{n}\right\|=\left\|\left[T\left(I-P_{M}\right)\right]^{n}\right\|=\left\|\left(T-P_{M}\right)^{n}\right\|=\left\|T^{n}-P_{M}\right\| .
$$

Now fix any $x \in X$ and set $y=x-P_{M} x$. Then $y \in M^{\perp}$ and

$$
\begin{aligned}
\left\|T^{n} x-P_{M} x\right\| & =\left\|T^{n}\left(x-P_{M} x\right)\right\|=\left\|T^{n} y\right\|=\left\|T^{n} P_{M^{\perp}} y\right\|=\left\|\left(T P_{M^{\perp}}\right)^{n} y\right\| \\
& \leq c_{n}(T)\|y\|=c_{n}(T)\left\|x-P_{M} x\right\|,
\end{aligned}
$$

which proves (3.14.1). 
(2) This was essentially proved during the course of proving (1).

(3) $c_{n}(T)=\left\|\left(T P_{M^{\perp}}\right)^{n}\right\| \leq\left\|T P_{M^{\perp}}\right\|^{n}=c_{1}(T)^{n}$.

(4) Let $N=\operatorname{Fix}\left(T^{*} T\right)$. Since $M=\operatorname{Fix} T^{*}$ by Lemma 2.1, it follows that $M \subset N$ and so $N^{\perp} \subset M^{\perp}$. Hence, since $T$ commutes with $P_{M^{\perp}}$ by Lemma 3.12 and, by a similar proof, $T^{*}$ commutes with $P_{M^{\perp}}$, we obtain

$$
\begin{aligned}
c\left(T^{*} T\right) & =\left\|T^{*} T P_{N^{\perp}}\right\| \leq\left\|T^{*} T P_{M^{\perp}}\right\|=\left\|\left(T P_{M^{\perp}}\right)^{*}\left(T P_{M^{\perp}}\right)\right\| \\
& =\left\|T P_{M^{\perp}}\right\|^{2}=c(T)^{2} .
\end{aligned}
$$

Moreover, if $\operatorname{Fix}\left(T^{*} T\right)=\operatorname{Fix} T$, then $N=M$ and $N^{\perp}=M^{\perp}$. So the above inequality must be an equality. Equation (3.14.2) holds when $T$ is a product of projections by Lemma 2.4 .

(5) Fix $x \in X$. Then $x-P_{M} x \in M^{\perp}$. So Lemma 3.11 and part (1) imply

$$
\left\|A_{T}(x)-P_{M} x\right\|=f(x)\left\|T x-P_{M} x\right\| \leq f(x) c(T)\left\|x-P_{M} x\right\| .
$$

Remark. The following example shows that the strict inequality $c\left(T^{*} T\right)<c(T)^{2}$ is possible in part (4). For let $X$ denote the Euclidean plane and define the linear operator $T$ on $X$ by $T x=x(2) e_{1}+x(1) e_{2}$ for each $x=(x(1), x(2)) \in X$, where $e_{1}=(1,0)$ and $e_{2}=(0,1)$. It is easy to verify that $\|T\|=1, M:=\operatorname{Fix} T=$ $\operatorname{span}\left(e_{1}+e_{2}\right), c(T)=1, T=T^{*}, T^{*} T=I$, and $c\left(T^{*} T\right)=c(I)=0$.

Lemma 3.15. Let $T$ be nonexpansive and $M=\operatorname{Fix} T$. Then

(1) if $T$ is normal, then $\left\|T^{n}-P_{M}\right\|=c(T)^{n}$ for every $n$;

(2) if $\operatorname{Fix}\left(T^{*} T\right)=\operatorname{Fix} T$, then

$$
\left\|\left(T^{*} T\right)^{n}-P_{M}\right\|=c(T)^{2 n} \quad \text { for every } n \in \mathbb{N} .
$$

In particular, for every $n \in \mathbb{N}$,

$$
\left\|\left(P_{M_{1}} P_{M_{2}} \cdots P_{M_{k}} P_{M_{k-1}} \cdots P_{M_{1}}\right)^{n}-P_{M}\right\|=c\left(M_{1}, M_{2}, \ldots, M_{k}\right)^{2 n} .
$$

Proof. (1) Since $T$ is normal and $T$ commutes with $P_{M^{\perp}}$ by Lemma 3.12, we deduce that $T P_{M \perp}$ is normal. Hence, using Lemma 3.14(1), we obtain

$$
\left\|T^{n}-P_{M}\right\|=c_{n}(T)=\left\|\left(T P_{M^{\perp}}\right)^{n}\right\|=\left\|T P_{M^{\perp}}\right\|^{n}=c(T)^{n} .
$$

(2) Since $T^{*} T$ is selfadjoint, hence normal, apply part (1) to $T^{*} T$ instead of $T$ using that Fix $\left(T^{*} T\right)=M$ to get $\left\|\left(T^{*} T\right)^{n}-P_{M}\right\|=c\left(T^{*} T\right)^{n}$. By Lemma 3.14(4), $c\left(T^{*} T\right)=c(T)^{2}$ and (3.15.1) follows.

(3.15.2) follows from (3.15.1) by taking $T=P_{M_{k}} P_{M_{k-1}} \cdots P_{M_{1}}$ and using Lemma 2.4 to get $\operatorname{Fix} T^{*} T=\operatorname{Fix} T$.

The following theorem gives an upper bound on the rate of convergence of the accelerated scheme.

Theorem 3.16. Let $x \in X$ and set

$$
x_{n}:=A_{T}^{n-1}(T x) \quad(n=1,2, \ldots) .
$$

Then for every $n \in \mathbb{N}$,

$$
\left\|T^{n} x-P_{M} x\right\| \leq c(T)^{n}\left\|x-P_{M} x\right\|
$$


and

$$
\left\|A_{T}^{n-1}(T x)-P_{M} x\right\| \leq\left[\prod_{1}^{n-1} f\left(x_{i}\right)\right] c(T)^{n}\left\|x-P_{M} x\right\| .
$$

Proof. The relation (3.16.1) is a consequence of Lemma 3.14(1) and (3).

We prove (3.16.2) by induction on $n$. For $n=1,\left\|T x-P_{M} x\right\| \leq c(T)\left\|x-P_{M} x\right\|$ by (3.14.1). Since the product of any set of scalars over the empty set of indices is 1 by definition, (3.16.2) holds when $n=1$. Now assume that (3.16.2) holds when $n=m \geq 1$. Then

$$
\begin{aligned}
\left\|A_{T}^{m}(T x)-P_{M} x\right\| & =\left\|x_{m+1}-P_{M} x\right\|=\left\|A_{T}\left(x_{m}\right)-P_{M} x\right\| \\
& =\left\|A_{T}\left(x_{m}\right)-P_{M}\left(x_{m}\right)\right\| \quad(\text { by Lemma 3.4) } \\
& =f\left(x_{m}\right)\left\|T\left(x_{m}\right)-P_{M}\left(x_{m}\right)\right\| \quad \text { by Lemma 3.11) } \\
& \leq f\left(x_{m}\right) c(T)\left\|x_{m}-P_{M}\left(x_{m}\right)\right\| \quad \text { by (3.14.1)) } \\
& =f\left(x_{m}\right) c(T)\left\|A_{T}^{m-1} T(x)-P_{M} x\right\| \\
& \leq f\left(x_{m}\right) c(T)\left[\prod_{i=1}^{m-1} f\left(x_{i}\right)\right] c(T)^{m}\left\|x-P_{M} x\right\| \\
& =\left[\prod_{1}^{m} f\left(x_{i}\right)\right] c(T)^{m+1}\left\|x-P_{M} x\right\|,
\end{aligned}
$$

which shows that (3.16.2) holds with $n$ replaced by $m+1$. This completes the induction.

Remarks. By comparing the right sides of (3.16.1) and (3.16.2), this result seems to suggest that the accelerated algorithm is always faster than its unaccelerated counterpart by at least the factor $\left[\prod_{1}^{n-1} f\left(x_{i}\right)\right]$. Indeed, we will show below that when $T$ is selfadjoint, nonnegative, and nonexpansive, then the accelerated method is faster than the original (see Theorem 3.20). In particular, the accelerated symmetric MAP is faster than the symmetric MAP. Also, the accelerated MAP for two subspaces is faster than the MAP. Perhaps surprisingly, however, we will see that this is not always the case, in general, for the accelerated MAP when there are more than two subspaces.

Theorem 3.16 can be strengthened in the particular case when $T=P_{2} P_{1}$. To do this, it is convenient to appeal to the following simple lemma (see, e.g., [13]).

Lemma 3.17. Let $M_{1}$ and $M_{2}$ be closed subspaces with $M=M_{1} \cap M_{2}$ and let $P_{i}$ be the orthogonal projection onto $M_{i}$ for $i=1,2$. Then $c\left(P_{2} P_{1}\right)=c\left(M_{1}, M_{2}\right)$ and

(1) if $x \in M_{1} \cap M^{\perp}$, then $\left\|P_{2} x\right\| \leq c\left(M_{1}, M_{2}\right)\|x\|$;

(2) if $x \in M_{2} \cap M^{\perp}$, then $\left\|P_{1} x\right\| \leq c\left(M_{1}, M_{2}\right)\|x\|$;

(3) if $x \in M_{2} \cap M^{\perp}$, then $\left\|P_{2} P_{1} x\right\| \leq c\left(M_{1}, M_{2}\right)^{2}\|x\|$.

Proof. That $c\left(P_{2} P_{1}\right)=c\left(M_{1}, M_{2}\right)$ in this case was observed following Definition 3.13 .

(1) Let $x \in M_{1} \cap M^{\perp}$. Then

$$
\left\|P_{2} x\right\|=\left\|P_{2} P_{1} P_{M^{\perp}} x\right\| \leq\left\|P_{2} P_{1} P_{M^{\perp}}\right\|\|x\|=c\left(P_{2} P_{1}\right)\|x\| .
$$

(2) The proof is similar to (1). 
(3) Let $x \in M_{2} \cap M^{\perp}$. Then $P_{1} x \in M_{1} \cap M^{\perp}$; so by (1) and (2), we obtain

$$
\left\|P_{2} P_{1} x\right\| \leq c\left(P_{2} P_{1}\right)\left\|P_{1} x\right\| \leq c\left(P_{2} P_{1}\right)^{2}\|x\|
$$

Theorem 3.18. Let $T=P_{M_{2}} P_{M_{1}}, x \in X$, and

$$
x_{n}:=A_{T}^{n-1}(T x) \quad(n=1,2, \ldots) .
$$

Then

$$
\left\|A_{T}^{n-1}(T x)-P_{M} x\right\| \leq\left[\prod_{1}^{n-1} f\left(x_{i}\right)\right] c\left(M_{1}, M_{2}\right)^{2 n-1}\left\|x-P_{M} x\right\| .
$$

Proof. The proof is by induction and proceeds just as in the proof of Theorem 3.16. The only point that should be noted is that in the induction step, we use the inequality $\left\|T\left(x_{m}\right)-P_{M}\left(x_{m}\right)\right\| \leq c(T)^{2}\left\|x_{m}-P_{M}\left(x_{m}\right)\right\|$ (rather than the same expression with $c(T)$ instead of $c(T)^{2}$ that was used in Theorem 3.16). The proof of this inequality follows immediately from Lemma 3.17(3).

Remarks. (1) Gearhart and Koshy [13] established (a weaker version of) the special case of Theorem 3.18 when $c:=c\left(M_{1}, M_{2}\right)<1$ and with an additional factor $\rho$ on the right side of (3.18.1), where $\rho:=\frac{1}{\sqrt{1-c^{2}}} \geq 1$.

(2) The inequality (3.18.1) improves the bound on the ordinary MAP in case $k=2$, due to Aronszajn [1], who showed that

$$
\left\|\left(P_{2} P_{1}\right)^{n} x-P_{M} x\right\| \leq c\left(M_{1}, M_{2}\right)^{2 n-1}\left\|x-P_{M} x\right\| \quad \text { for all } x \in X \text {. }
$$

In fact, Kayalar and Weinert [17] showed that the Aronszajn bound is sharp, i.e., $\left\|\left(P_{2} P_{1}\right)^{n}-P_{M}\right\|=c\left(M_{1}, M_{2}\right)^{2 n-1}$.

Next we show that the accelerated algorithms are always at least as fast as their unaccelerated counterparts provided that $T$ is selfadjoint, nonnegative, and nonexpansive. It is first convenient to establish the following result.

Lemma 3.19. If

$$
\left\|T^{n-1}\left(A_{T}(x)\right)\right\| \leq\left\|T^{n} x\right\| \quad \text { for every } x \in M^{\perp} \text { and } n \in \mathbb{N}
$$

then

$$
\left\|A_{T}^{n-1}(T x)\right\| \leq\left\|T^{n} x\right\| \quad \text { for every } x \in M^{\perp} \text { and } n \in \mathbb{N}
$$

In particular, if (3.19.1) holds and the original algorithm converges, then

$$
\left\|A_{T}^{n-1}(T x)-P_{M} x\right\| \leq\left\|T^{n} x-P_{M} x\right\| \quad \text { for every } x \in X, n \in \mathbb{N},
$$

and hence the accelerated algorithm converges at least as fast as the original. 
Proof. When $n=1,(3.19 .2)$ is trivial. If $n \geq 2$, then for each $x \in M^{\perp}$,

$$
\begin{aligned}
\left\|A_{T}^{n-1}(T x)\right\| & =\left\|A_{T}\left(A_{T}^{n-2}(T x)\right)\right\| \leq\left\|T\left(A_{T}^{n-2}(T x)\right)\right\| \quad \text { using }(3.7 .5) \\
& =\left\|T\left(A_{T}(y)\right)\right\|, \quad \text { where } y:=A_{T}^{n-3}(T x) \in M^{\perp} \text { by Lemma } 3.3(4) \\
& \leq\left\|T^{2} y\right\| \quad \text { by }(3.19 .1) \\
& =\left\|T^{2}\left(A_{T}^{n-3}(T x)\right)\right\| \\
& =\left\|T^{2}\left(A_{T}(z)\right)\right\|, \quad \text { where } z:=A_{T}^{n-4}(T x) \in M^{\perp} \text { by Lemma } 3.3(4) \\
& \leq\left\|T^{3} z\right\| \quad \text { by }(3.19 .1) \\
& =\left\|T^{3}\left(A_{T}^{n-4}(T x)\right)\right\| .
\end{aligned}
$$

Continuing in this way, we end up with the inequality $\left\|A_{T}^{n-1}(T x)\right\| \leq\left\|T^{n} x\right\|$, which verifies (3.19.2) when $n \geq 2$.

To verify the last statement, let $x \in X$. Then $x-P_{M} x \in M^{\perp}$ and so by (3.19.2) and Lemma 3.8(3), we get

$$
\left\|A_{T}^{n-1}(T x)-P_{M} x\right\|=\left\|A_{T}^{n-1}\left(T\left(x-P_{M} x\right)\right)\right\| \leq\left\|T^{n}\left(x-P_{M} x\right)\right\|=\left\|T^{n} x-P_{M} x\right\|
$$

and this verifies (3.19.3).

The natural question raised by Lemma 3.19 is this: for which $T$ does (3.19.1) hold? We will show next that if $T$ is selfadjoint, nonnegative, and nonexpansive, then (3.19.1) and hence (3.19.3) hold. It should be noted that our proof seems to use the spectral theorem (for compact selfadjoint operators) in an essential way.

Theorem 3.20. Let $T$ be selfadjoint, nonnegative, and nonexpansive. Then

$$
\left\|A_{T}^{n-1}(T x)-P_{M} x\right\| \leq\left\|T^{n} x-P_{M} x\right\| \quad \text { for each } x \in X \text { and } n \in \mathbb{N} .
$$

In other words, the accelerated algorithm converges at least as fast as its unaccelerated counterpart.

Corollary 3.21. If $T=P_{1} P_{2} \cdots P_{k} P_{k-1} \cdots P_{1}$, then

$$
\left\|A_{T}^{n-1}(T x)-P_{M} x\right\| \leq\left\|T^{n} x-P_{M} x\right\| \quad \text { for each } x \in X \text { and } n \in \mathbb{N} .
$$

In other words, the accelerated symmetric MAP is at least as fast as the symmetric $M A P$.

The corollary follows since $T=Q^{*} Q$, where $Q=P_{k} P_{k-1} \cdots P_{1}$.

Proof of Theorem 3.20. By Lemma 3.19, it suffices to show that

$$
\left\|T^{m-1} A_{T}(y)\right\| \leq\left\|T^{m} y\right\| \quad \text { for every } y \in M^{\perp} \text { and } m \in \mathbb{N} .
$$

Toward this end, fix $y \in M^{\perp}$ and $m \in \mathbb{N}$. If $y=0$, (3.20.2) is trivial. Thus, by scaling and Lemma 3.8(4), we may assume $\|y\|=1$. If $m=1$, then (3.20.2) follows from (3.7.5). Thus, we may assume $m \geq 2$. Let

$$
N=\operatorname{span}\left\{y, T y, T^{2} y, \ldots, T^{m} y\right\}
$$

By Lemma 3.3(4), $N \subset M^{\perp}$. Define $S:=P_{N} T P_{N}$. Then $S$ is compact, selfadjoint, nonexpansive, $\mathcal{R}(S):=$ Range of $S \subset N$, and so $n:=\operatorname{dim} \mathcal{R}(S) \leq m+1$. We may assume that $T y \neq 0$. For if $T y=0$, then $A_{T}(y)=0$ by (3.7.5); so (3.20.2) holds and we are done. But if $T y \neq 0$, then $S y \neq 0$ and hence $n \geq 1$. As a consequence of 
the Spectral Theorem [3, Corollary 5.4, p. 47], we readily deduce that there exists an orthonormal set of $n$ eigenvectors $\left\{v_{1}, v_{2}, \ldots, v_{n}\right\}$ of $S$ such that

$$
S x:=\sum_{1}^{n} \lambda_{i}\left\langle x, v_{i}\right\rangle v_{i} \text { for every } x \in X
$$

where $\lambda_{i}$ is the (nonzero) eigenvalue corresponding to $v_{i}: S v_{i}=\lambda_{i} v_{i} \quad(i=$ $1,2, \ldots, n)$. In particular, $\left\{v_{1}, \ldots, v_{n}\right\}$ is an orthonormal basis for $\mathcal{R}(S)$. Since $T$ is nonnegative,

$$
\begin{aligned}
\lambda_{i} & =\lambda_{i}\left\langle v_{i}, v_{i}\right\rangle=\left\langle\lambda_{i} v_{i}, v_{i}\right\rangle=\left\langle S v_{i}, v_{i}\right\rangle=\left\langle P_{N} T P_{N} v_{i}, v_{i}\right\rangle \\
& =\left\langle T P_{N} v_{i}, P_{N} v_{i}\right\rangle=\left\langle T v_{i}, v_{i}\right\rangle \quad \text { since } v_{i} \in N \\
& \geq 0 .
\end{aligned}
$$

Thus, $\lambda_{i}>0$ for each $i$. Since $S$ is nonexpansive,

$$
\lambda_{i}=\left\|\lambda_{i} v_{i}\right\|=\left\|S v_{i}\right\| \leq\left\|v_{i}\right\|=1 .
$$

We have shown that $0<\lambda_{i} \leq 1$ for each $i$. Moreover, if some $\lambda_{i}=1$, then

$$
\begin{aligned}
1 & =\left\langle v_{i}, v_{i}\right\rangle=\left\langle v_{i}, S v_{i}\right\rangle=\left\langle v_{i}, P_{N} T P_{N} v_{i}\right\rangle \\
& =\left\langle v_{i}, T P_{N} v_{i}\right\rangle=\left\langle v_{i}, T v_{i}\right\rangle \leq\left\|v_{i}\right\|\left\|T v_{i}\right\| \leq 1 .
\end{aligned}
$$

So equality must hold throughout this string of inequalities. Using the condition of equality in Schwarz's inequality, we obtain $T v_{i}=\rho v_{i}$ for some $\rho>0$ and $\left\|T v_{i}\right\|=$ $\left\|v_{i}\right\|=1$. Hence, $\rho=1$ and $T v_{i}=v_{i}$. That is, $v_{i} \in \operatorname{Fix} T=M$. But $v_{i} \in M^{\perp}$ implies that $v_{i}=0$, a contradiction. This proves that $\lambda_{i}<1$ for each $i$. Hence, we have shown that

$$
0<\lambda_{i}<1 \quad \text { for } i=1,2, \ldots, n .
$$

Let $\alpha_{i}:=\left\langle y, v_{i}\right\rangle$ for each $i$.

Claim 1. $T^{j} y=S^{j} y=\sum_{i=1}^{n} \alpha_{i} \lambda_{i}^{j} v_{i} \quad(j=1,2, \ldots, m)$.

The formula for $S, S^{j} y=\sum_{1}^{n} \alpha_{i} \lambda_{i}^{j} v_{i}$, follows easily from (3.20.3) and the fact that $S v_{i}=\lambda_{i} v_{i}$. To prove the corresponding statement about $T$, we proceed by induction on $j$. For $j=1$, since $y$ and $T y$ are in $N$, we obtain $T y=P_{N} T y=$ $P_{N} T P_{N} y=S y$; so the result holds when $j=1$. Now suppose the result holds when $j=l \leq m-1$. Then

$$
S^{l+1} y=S\left(S^{l} y\right)=S\left(T^{l} y\right)=P_{N} T P_{N}\left(T^{l} y\right)=P_{N} T\left(T^{l} y\right)=P_{N} T^{l+1} y=T^{l+1} y
$$

since $T^{l+1} y \in N$. This proves the claim.

Since $\mathcal{R}(S)^{\perp}=\mathcal{N}\left(S^{*}\right)=\mathcal{N}(S)$, where $\mathcal{N}(S)$ is the null space of $S$, we have that $X=\mathcal{R}(S) \oplus \mathcal{N}(S)$ and hence we can write $y$ as $y=y_{1}+y_{0}$, where $y_{1} \in \mathcal{R}(S)=$ $\operatorname{span}\left\{v_{1}, v_{2}, \ldots, v_{n}\right\}$ and $y_{0} \in \operatorname{span}\left\{v_{1}, v_{2}, \ldots, v_{n}\right\}^{\perp}=\mathcal{N}(S)$. Then

$$
y=\sum_{1}^{n}\left\langle y_{1}, v_{i}\right\rangle v_{i}+y_{0}=\sum_{1}^{n}\left\langle y, v_{i}\right\rangle v_{i}+y_{0}=\sum_{1}^{n} \alpha_{i} v_{i}+y_{0}
$$

and

$$
\|y\|^{2}=\sum_{1}^{n} \alpha_{i}^{2}+\left\|y_{0}\right\|^{2}
$$

Claim 2. $T^{m-1} A_{T}(y)=\sum_{i=1}^{n} \alpha_{i} \lambda_{i}^{m-1}\left\{1-\left(1-\lambda_{i}\right) t_{y}\right\} v_{i}$. 
We compute

$$
\begin{aligned}
T^{m-1} A_{T}(y) & =T^{m-1}\left[t_{y} T y+\left(1-t_{y}\right) y\right]=t_{y} T^{m} y+\left(1-t_{y}\right) T^{m-1} y \\
& =t_{y} S^{m} y+\left(1-t_{y}\right) S^{m-1} y=t_{y} \sum_{1}^{n} \alpha_{i} \lambda_{i}^{m} v_{i}+\left(1-t_{y}\right) \sum_{1}^{n} \alpha_{i} \lambda_{i}^{m-1} v_{i} \\
& =\sum_{1}^{n} \alpha_{i} \lambda_{i}^{m-1}\left\{t_{y} \lambda_{i}+\left(1-t_{y}\right)\right\} v_{i}=\sum_{1}^{n} \alpha_{i} \lambda_{i}^{m-1}\left\{1-\left(1-\lambda_{i}\right) t_{y}\right\} v_{i}
\end{aligned}
$$

which proves the claim.

By Claims 1 and 2, we see that (3.20.2) holds if and only if

$$
\sum_{i=1}^{n} \alpha_{i}^{2} \lambda_{i}^{2 m-2}\left\{1-\left(1-\lambda_{i}\right) t_{y}\right\}^{2} \leq \sum_{i=1}^{n} \alpha_{i}^{2} \lambda_{i}^{2 m}
$$

which, after some algebra, may be rewritten as

$$
q\left(t_{y}\right) \leq 0
$$

where

$$
\begin{array}{rlrl}
q(t) & :=\alpha t^{2}-2 \beta t+\gamma, & \alpha:=\sum_{1}^{n} \alpha_{i}^{2} \lambda_{i}^{2 m-2}\left(1-\lambda_{i}\right)^{2}, \\
\beta:=\sum_{1}^{n} \alpha_{i}^{2} \lambda_{i}^{2 m-2}\left(1-\lambda_{i}\right), & \gamma:=\sum_{1}^{n} \alpha_{i}^{2} \lambda_{i}^{2 m-2}\left(1-\lambda_{i}^{2}\right) .
\end{array}
$$

Claim 3. The function $h$, defined on the nonnegative real line by

$$
h(t):=\frac{\sum_{i} \alpha_{i}^{2} \lambda_{i}^{t}\left(1-\lambda_{i}\right)}{\sum_{j} \alpha_{j}^{2} \lambda_{j}^{t}\left(1-\lambda_{j}\right)^{2}} \quad \text { for all } t \geq 0
$$

is increasing.

Writing $h(t)=u(t) / v(t)$, it suffices to verify that $h^{\prime}(t) \geq 0$. Equivalently, it suffices to show that

$$
u^{\prime}(t) v(t) \geq u(t) v^{\prime}(t) \quad \text { for all } t \geq 0 .
$$

Setting

$$
\beta_{i}=\frac{\alpha_{i}^{2}\left(1-\lambda_{i}\right) \lambda_{i}^{t}}{\sum_{j} \alpha_{j}^{2}\left(1-\lambda_{j}\right) \lambda_{j}^{t}},
$$

we see that $\beta_{i} \geq 0, \sum_{1}^{n} \beta_{i}=1$, and (3.20.7) may be rewritten as

$$
\sum_{j} \beta_{j} \lambda_{j} \ln \lambda_{j} \geq\left(\sum_{i} \beta_{i} \ln \lambda_{i}\right)\left(\sum_{j} \beta_{j} \lambda_{j}\right) .
$$

Since the function $t \mapsto t \ln t$ is convex on $(0, \infty)$, it follows that

$$
\left(\sum_{j} \beta_{j} \lambda_{j}\right) \ln \left(\sum_{i} \beta_{i} \lambda_{i}\right) \leq \sum_{j} \beta_{j} \lambda_{j} \ln \lambda_{j} .
$$

On the other hand, the function $t \mapsto \ln t$ is concave on $(0, \infty)$; so

$$
\ln \left(\sum_{j} \beta_{j} \lambda_{j}\right) \geq \sum_{j} \beta_{j} \ln \lambda_{j} .
$$


Combining (3.20.9) and (3.20.10), we obtain (3.20.8), and this proves Claim 3.

To prove (3.20.5), and finish the proof of the theorem, we must verify that $q\left(t_{y}\right) \leq 0$, where $q$ is the quadratic defined in (3.20.6). Now $q(0)=\gamma>0$ and $q(1)=\alpha-2 \beta+\gamma=0$. Also, an inspection of the coefficients shows that $0<\alpha<$ $\beta<\gamma$. Further, the quadratic formula shows that the zeros of $q$ are given by

$$
t_{\min }=\frac{\beta-\sqrt{\beta^{2}-\alpha \gamma}}{\alpha}, \quad \text { and } t_{\max }=\frac{\beta+\sqrt{\beta^{2}-\alpha \gamma}}{\alpha} .
$$

Since $\beta=\frac{1}{2}(\alpha+\gamma)$, it follows that $t_{\min }=1$ and $t_{\max }=\gamma / \alpha>1$. Since $q$ has a positive leading coefficient, we see that $q(t) \leq 0$ if and only if $t_{\min } \leq t \leq t_{\max }$, i.e., $1 \leq t \leq \gamma / \alpha$. Thus to prove $q\left(t_{y}\right) \leq 0$, we must show that

$$
1 \leq t_{y} \leq \frac{\gamma}{\alpha}
$$

We have, using Claim 1, that

$$
\begin{aligned}
t_{y} & =\frac{\langle y, y-T y\rangle}{\|y-T y\|^{2}}=\frac{\left\langle\sum_{i} \alpha_{i} v_{i}+y_{0}, \sum_{i} \alpha_{i}\left(1-\lambda_{i}\right) v_{i}+y_{0}\right\rangle}{\sum_{i} \alpha_{i}^{2}\left(1-\lambda_{i}\right)^{2}+\left\|y_{0}\right\|^{2}} \\
& =\frac{\sum_{i} \alpha_{i}^{2}\left(1-\lambda_{i}\right)+\left\|y_{0}\right\|^{2}}{\sum_{i} \alpha_{i}^{2}\left(1-\lambda_{i}\right)^{2}+\left\|y_{0}\right\|^{2}} .
\end{aligned}
$$

Since $0<\left(1-\lambda_{i}\right)^{2}<1-\lambda_{i}$, it follows that $t_{y} \geq 1$. Also, $t_{y} \leq \gamma / \alpha$ is equivalent to

$$
\frac{\sum_{i} \alpha_{i}^{2}\left(1-\lambda_{i}\right)+\left\|y_{0}\right\|^{2}}{\sum_{j} \alpha_{j}^{2}\left(1-\lambda_{j}\right)^{2}+\left\|y_{0}\right\|^{2}} \leq \frac{\sum_{i} \alpha_{i}^{2} \lambda_{i}^{2 m-2}\left(1-\lambda_{i}^{2}\right)}{\sum_{j} \alpha_{j}^{2} \lambda_{j}^{2 m-2}\left(1-\lambda_{j}\right)^{2}} \text {. }
$$

But

$$
\frac{\sum_{i} \alpha_{i}^{2}\left(1-\lambda_{i}\right)+\left\|y_{0}\right\|^{2}}{\sum_{j} \alpha_{j}^{2}\left(1-\lambda_{j}\right)^{2}+\left\|y_{0}\right\|^{2}} \leq \frac{\sum_{i} \alpha_{i}^{2}\left(1-\lambda_{i}\right)}{\sum_{j} \alpha_{j}^{2}\left(1-\lambda_{j}\right)^{2}}
$$

follows since $\sum_{i} \alpha_{i}^{2}\left(1-\lambda_{i}\right) \geq \sum_{i} \alpha_{i}^{2}\left(1-\lambda_{i}\right)^{2}$.

By Claim 3, $h$ is increasing so that $h(0) \leq h(2 m-2)$. That is,

$$
\frac{\sum_{i} \alpha_{i}^{2}\left(1-\lambda_{i}\right)}{\sum_{j} \alpha_{j}^{2}\left(1-\lambda_{j}\right)^{2}} \leq \frac{\sum_{i} \alpha_{i}^{2} \lambda_{i}^{2 m-2}\left(1-\lambda_{i}\right)}{\sum_{j} \alpha_{j}^{2} \lambda_{j}^{2 m-2}\left(1-\lambda_{j}\right)^{2}}
$$

Combining (3.20.13) and (3.20.14), we obtain (3.20.12) and hence $t_{y} \leq \gamma / \alpha$. This proves (3.20.12), and completes the proof of the theorem.

A certain analogue of Theorem 3.20, valid when $T$ is not selfadjoint, can be deduced from Theorem 3.20 as follows.

Corollary 3.22. Suppose $S$ is a bounded linear operator on $X, L$ is a closed subspace of $X$ such that $L \supset \mathcal{R}(S)$, and $S P_{L}$ is selfadjoint, nonnegative, and nonexpansive. Let $M=\operatorname{Fix} S$. Then

$$
\left\|A_{S}^{n-1} S P_{L} x-P_{M} x\right\| \leq\left\|S^{n} P_{L} x-P_{M} x\right\| \quad \text { for each } x \in X \text { and } n \in \mathbb{N} .
$$

In particular,

$$
\left\|A_{S}^{n-1} S x-P_{M} x\right\| \leq\left\|S^{n} x-P_{M} x\right\| \quad \text { for each } x \in L \text { and } n \in \mathbb{N} .
$$

Proof. Set $T=S P_{L}$. Then $T$ satisfies the hypothesis of Theorem 3.20. Moreover, since $\mathcal{R}(S) \subset L$, it follows that Fix $T=$ Fix $S=M$. Thus, we deduce from (3.20.1) that

$$
\left\|A_{T}^{n-1}(T x)-P_{M} x\right\| \leq\left\|T^{n} x-P_{M} x\right\| \quad \text { for each } x \in X \text { and } n \in \mathbb{N} \text {. }
$$


Since $\mathcal{R}(S) \subset L$, we deduce that

$$
T^{n}=\left(S P_{L}\right)^{n}=S\left(P_{L} S\right)^{n-1} P_{L}=S(S)^{n-1} P_{L}=S^{n} P_{L} .
$$

In particular, $T^{n} x=S^{n} x$ for each $x \in L$. Moreover, for each $y \in L$,

$$
A_{T}(y)=t_{y, T} T y+\left(1-t_{y, T}\right) y=t_{y, T} S y+\left(1-t_{y, T}\right) y
$$

and $A_{S} y=t_{y, S} S y+\left(1-t_{y, S}\right) y$. But

$$
t_{y, T}=\frac{\langle y, y-T y\rangle}{\|y-T y\|^{2}}=\frac{\langle y, y-S y\rangle}{\|y-S y\|^{2}}=t_{y, S} ;
$$

so $A_{T}(y)=A_{S} y \in L$ so that, inductively, $A_{T}^{n-1}(y)=A_{S}^{n-1} y$. Substituting back into (3.22.3), we obtain (3.22.2). In general, for any $y \in X, x=P_{L} y \in L$, and so

$$
\left\|A_{S}^{n-1} S P_{L} y-P_{M} P_{L} y\right\| \leq\left\|S^{n} P_{L} y-P_{M} P_{L} y\right\| \text {. }
$$

But $M \subset \mathcal{R}(S) \subset L$; so $P_{M} P_{L} y=P_{M} y$ and substituting this into (3.22.4) yields (3.22.1).

One application of Corollary 3.22 is in the case of the MAP for two subspaces.

Theorem 3.23. Let $M_{1}$ and $M_{2}$ be closed subspaces in $X, Q=P_{2} P_{1}$, and $M=$ $M_{1} \cap M_{2}$. Then for each $n \in \mathbb{N}$,

$$
\left\|A_{Q}^{n-1} Q x-P_{M} x\right\| \leq\left\|Q^{n} x-P_{M} x\right\| \quad \text { for every } x \in X .
$$

In other words, the accelerated MAP is faster than the MAP in the case of two subspaces.

Proof. Take $S=Q$ and $L=M_{2}$ in Corollary 3.22 to obtain

$$
\left\|A_{Q}^{n-1} Q P_{2} x-P_{M} x\right\| \leq\left\|Q^{n} P_{2} x-P_{M} x\right\| \quad \text { for every } x \in X \text {. }
$$

In particular, (3.23.1) holds for each $x \in M_{2}$. It remains to show that (3.23.1) holds for all $x \in X$. We first verify

$$
\overline{\mathcal{R}\left(P_{2} P_{1} P_{2}\right)}=\overline{\mathcal{R}\left(P_{2} P_{1}\right)} .
$$

To see this, note that it is well-known that for any bounded linear operator $T$ on $X$,

$$
\mathcal{N}\left(T^{*} T\right)=\mathcal{N}(T) \quad \text { and } \quad \mathcal{N}(T)^{\perp}=\overline{\mathcal{R}\left(T^{*}\right)} .
$$

Putting $T=P_{1} P_{2}$ in (3.23.4), we obtain that $\mathcal{N}\left(P_{2} P_{1} P_{2}\right)=\mathcal{N}\left(P_{1} P_{2}\right)$ and hence $\overline{\mathcal{R}\left(P_{2} P_{1} P_{2}\right)}=\mathcal{N}\left(P_{2} P_{1} P_{2}\right)^{\perp}=\mathcal{N}\left(P_{1} P_{2}\right)^{\perp}=\overline{\mathcal{R}\left(P_{2} P_{1}\right)}$, which proves $(3.23 .3)$.

Now fix any $x \in X$ and set $z=P_{2} P_{1} x$. Then $z \in \mathcal{R}\left(P_{2} P_{1}\right)$ and so, by (3.23.3), we obtain that $z=\lim z_{k}$, where $z_{k} \in \mathcal{R}\left(P_{2} P_{1} P_{2}\right)$ for each $k$. Then we choose $w_{k} \in X$ so that $z_{k}=P_{2} P_{1} P_{2} w_{k}$. Let $y_{k}:=P_{2} w_{k}$. Then $y_{k} \in M_{2}$ and $z_{k}=P_{2} P_{1} y_{k}$. Since $P_{i}$ commutes with $P_{M}$ for $i=1,2$, we have that

$$
\begin{aligned}
P_{M} x & =P_{2} P_{1} P_{M} x=P_{M} P_{2} P_{1} x=P_{M} z=\lim _{k} P_{M} z_{k} \\
& =\lim _{k} P_{M} P_{2} P_{1} y_{k}=\lim _{k} P_{2} P_{1} P_{M} y_{k}=\lim _{k} P_{M} y_{k} .
\end{aligned}
$$

Moreover,

$$
\lim _{k} Q y_{k}=\lim _{k} P_{2} P_{1} y_{k}=\lim _{k} z_{k}=z=Q x .
$$

By (3.23.2) applied to $y_{k} \in M_{2}$, we obtain that

$$
\left\|A_{Q}^{n-1} Q y_{k}-P_{M} y_{k}\right\| \leq\left\|Q^{n} y_{k}-P_{M} y_{k}\right\| .
$$


Letting $k \rightarrow \infty$ in (3.23.7), and using (3.23.5), (3.23.6), and the continuity of $A_{Q}$ (Lemma 3.8(5)), we obtain (3.23.1).

The following is an example showing that the accelerated MAP may be slower than the MAP when there are more than two subspaces!

Example 3.24. Let $X=\ell_{2}$ and let $e_{i}(i=1,2, \ldots)$ denote the canonical unit vectors in $X: e_{i}(j)=\delta_{i j}$ for all $i, j$. Define five 2-dimensional subspaces as follows:

$$
\begin{aligned}
& M_{1}=\operatorname{span}\left\{e_{2}, e_{3}\right\}, \quad M_{2}=\operatorname{span}\left\{e_{2}+e_{4}, e_{3}+e_{5}\right\}, \quad M_{3}=\operatorname{span}\left\{e_{4}, e_{5}\right\}, \\
& M_{4}=\operatorname{span}\left\{e_{1}+e_{4}, e_{2}+e_{5}\right\}, \quad \text { and } \quad M_{5}=\operatorname{span}\left\{e_{1}, e_{2}\right\} .
\end{aligned}
$$

Let $P_{i}=P_{M_{i}}$ for $i=1,2, \ldots, 5$ and $T:=P_{5} P_{4} P_{3} P_{2} P_{1}$. It is easy to verify that

$$
T x=\frac{1}{4} x(2) e_{1}+\frac{1}{4} x(3) e_{2} \quad \text { for each } x \in \ell_{2} .
$$

Also, $\|T\|=\frac{1}{4}$ and $M:=\operatorname{Fix} T=\{0\}$. Set $x_{0}:=4 e_{3}$. Then $T x_{0}=e_{2}, T^{2} x_{0}=\frac{1}{4} e_{1}$, and $T^{n} x_{0}=0$ for all $n \geq 3$.

Let $z_{0}:=T x_{0}=e_{2}$ and define $z_{n}:=A_{T}\left(z_{n-1}\right)=A_{T}^{n}\left(z_{0}\right)$ for $n \geq 1$. Since the range of $T$ is span $\left\{e_{1}, e_{2}\right\}$ and $A_{T}(x)$ is an affine combination of $T x$ and $x$, it follows that

$$
z_{n}=\alpha_{n} e_{1}+\beta_{n} e_{2} \quad(n=0,1, \ldots)
$$

for some scalars $\alpha_{n}, \beta_{n}$. We will prove that $z_{n} \neq 0$ for every $n$.

Having done this, we would then obtain for every $n \geq 3$ that

$\left\|A_{T}^{n-1}\left(T x_{0}\right)-P_{M} x_{0}\right\|=\left\|A_{T}^{n-1}\left(T x_{0}\right)\right\|=\left\|z_{n-1}\right\|>0=\left\|T^{n} x_{0}\right\|=\left\|T^{n} x_{0}-P_{M} x_{0}\right\|$ which shows that the accelerated MAP is slower than the MAP beginning with the third iterate. (It should be noted, however, that the second iterate for the accelerated method has a strictly smaller norm than the corresponding unaccelerated term: $\left\|A_{T}\left(T x_{0}\right)\right\|=1 / \sqrt{17}<1 / 4=\left\|T^{2} x_{0}\right\|$.)

It remains to show that $z_{n} \neq 0$ for each $n$, and this will be done through a series of claims.

Claim 1. $T z_{n}=\frac{1}{4} \beta_{n} e_{1}(n=0,1, \ldots)$.

This follows from

$$
T z_{n}=T\left(\alpha_{n} e_{1}+\beta_{n} e_{2}\right)=\alpha_{n} T e_{1}+\beta_{n} T e_{2}=\frac{1}{4} \beta_{n} e_{1} .
$$

Next we prove

Claim 2. $z_{n+1}=0$ if and only if $\beta_{n}=0$.

For suppose $z_{n+1}=0$. Then

$$
\begin{aligned}
0 & =A_{T}\left(z_{n}\right)=t_{n} T z_{n}+\left(1-t_{n}\right) z_{n}, \text { where } t_{n}=t_{z_{n}} \\
& =\frac{1}{4} \beta_{n} t_{n} e_{1}+\left(1-t_{n}\right)\left(\alpha_{n} e_{1}+\beta_{n} e_{2}\right) \\
& =\left[\frac{1}{4} \beta_{n} t_{n}+\left(1-t_{n}\right) \alpha_{n}\right] e_{1}+\left(1-t_{n}\right) \beta_{n} e_{2} .
\end{aligned}
$$

It follows that

$$
\frac{1}{4} \beta_{n} t_{n}+\left(1-t_{n}\right) \alpha_{n}=0 \quad \text { and } \quad\left(1-t_{n}\right) \beta_{n}=0 .
$$

No matter what the value of $t_{n}$ is, the two equations above imply $\beta_{n}=0$.

Conversely, if $\beta_{n}=0$, then $z_{n}=\alpha_{n} e_{1}$ implies that $T z_{n}=0$ and hence $A_{T}\left(z_{n}\right)=$ 0 (since $\left\|A_{T}\left(z_{n}\right)\right\| \leq\left\|T z_{n}\right\|$ by (3.7.5)). Thus, $z_{n+1}=A_{T}\left(z_{n}\right)=0$. 
Claim 3. For $n=0,1,2, \ldots$,

$$
\beta_{n+1} \beta_{n}=\alpha_{n+1}\left(\frac{1}{4} \beta_{n}-\alpha_{n}\right) .
$$

In particular, if $\beta_{n} \neq 0$, then

$$
\beta_{n+1}=\alpha_{n+1}\left(\frac{1}{4}-\frac{\alpha_{n}}{\beta_{n}}\right) .
$$

To verify this, note that by Lemma 3.3(2), we obtain that

$$
\left\langle z_{n+1}, z_{n}-T z_{n}\right\rangle=\left\langle A_{T}\left(z_{n}\right), z_{n}-T z_{n}\right\rangle=0 .
$$

Using the representation of $z_{n}$ in (3.24.1), we expand the above equation and deduce that $\alpha_{n+1}\left(\alpha_{n}-\frac{1}{4} \beta_{n}\right)+\beta_{n+1} \beta_{n}=0$, which is just (3.24.2).

If the result that $z_{n} \neq 0$ for each $n$ were false, we let $n_{0}$ denote the smallest integer such that $z_{n_{0}+1}=0$. Now $\beta_{0}=1$ and one can readily compute that

$$
z_{1}=A_{T}\left(z_{0}\right)=A_{T}\left(e_{2}\right)=t_{e_{2}} T e_{2}+\left(1-t_{e_{2}}\right) e_{2}=\frac{1}{4} t_{e_{2}} e_{1}+\left(1-t_{e_{2}}\right) e_{2},
$$

where

$$
t_{e_{2}}=\frac{\left\langle e_{2}, e_{2}-T e_{2}\right\rangle}{\left\|e_{2}-T e_{2}\right\|^{2}}=\frac{1}{\left\|e_{2}-\frac{1}{4} e_{1}\right\|^{2}}=\frac{16}{17} .
$$

Hence, $z_{1}=\frac{4}{17} e_{1}+\frac{1}{17} e_{2}$ and so $\alpha_{1}=\frac{4}{17}$ and $\beta_{1}=\frac{1}{17}$. Thus $\beta_{0} \neq 0$ and $\beta_{1} \neq 0$. By Claim 2, $\beta_{n_{0}}=0$; so $n_{0} \geq 2$, and $\beta_{n} \neq 0$ for every $n \leq n_{0}-1$. Further, by Claim 3, we deduce

$$
\beta_{n+1}=\alpha_{n+1}\left(\frac{1}{4}-\mu_{n}\right) \quad \text { for } n=0,1, \ldots, n_{0}-1,
$$

where $\mu_{n}:=\alpha_{n} / \beta_{n}$.

From (3.24.4), we deduce that $\alpha_{n+1} \neq 0$ whenever $\beta_{n+1} \neq 0$ and $0 \leq n \leq n_{0}-1$. Since $\beta_{k+1} \neq 0$ for $0 \leq k \leq n_{0}-2$, it follows that $\alpha_{k+1} \neq 0$ for $0 \leq k \leq n_{0}-2$. In other words,

$$
\alpha_{n} \neq 0 \quad \text { and } \quad \beta_{n} \neq 0 \quad \text { for } \quad 1 \leq n \leq n_{0}-1 .
$$

Using (3.24.4), we obtain that

$$
0 \neq \frac{\beta_{n+1}}{\alpha_{n+1}}=\frac{1}{4}-\mu_{n} \quad \text { for } \quad 0 \leq n \leq n_{0}-2 .
$$

Next consider the following subset of the rational numbers:

$$
\mathbb{Q}^{*}:=\left\{\frac{p}{q} \mid p, q \in \mathbb{Z}, p \text { even, } q \text { odd }\right\} .
$$

In particular, $0 \in \mathbb{Q}^{*}$ but $\frac{1}{4} \notin \mathbb{Q}^{*}$.

Claim 4. The function $f(x)=\left(\frac{1}{4}-x\right)^{-1}$ maps $\mathbb{Q}^{*}$ into $\mathbb{Q}^{*}$.

First, note that $f$ is well-defined since $\frac{1}{4} \notin \mathbb{Q}^{*}$. Next, let $x \in \mathbb{Q}^{*}$. Then $x=\frac{p}{q}$ for some even $p$ and odd $q$. Hence,

$$
f(x)=\frac{1}{\frac{1}{4}-\frac{p}{q}}=\frac{4 q}{q-4 p} .
$$

Since $4 q$ is even and $q-4 p$ is odd, it follows that $f(x) \in \mathbb{Q}^{*}$.

Claim 5. $\mu_{n} \in \mathbb{Q}^{*}$ for $0 \leq n \leq n_{0}-1$. In particular, $\mu_{n} \neq \frac{1}{4}$ for $0 \leq n \leq n_{0}-1$. 
To verify this, first note that $\mu_{0}=\frac{\alpha_{0}}{\beta_{0}}=0 \in \mathbb{Q}^{*}$. By (3.24.6), it follows that

$$
\mu_{n+1}:=\frac{\alpha_{n+1}}{\beta_{n+1}}=\frac{1}{\frac{1}{4}-\mu_{n}} \quad\left(n=0,1, \ldots, n_{0}-2\right) .
$$

Using (3.24.7), Claim 4, and induction, it follows that $\mu_{n+1} \in \mathbb{Q}^{*}$ for $n=$ $0,1, \ldots, n_{0}-2$. This proves Claim 5 .

Finally, $\mu_{n_{0}-1} \neq \frac{1}{4}$ from Claim 5. Since $\beta_{n_{0}}=0,(3.24 .4)$ implies that $\alpha_{n_{0}}=0$. But then $z_{n_{0}}=\alpha_{n_{0}} e_{1}+\beta_{n_{0}} e_{2}=0$, which contradicts the choice of $n_{0}$. This proves that the accelerated MAP is slower than the MAP for this example. However, both the MAP and the accelerated MAP do converge! This raises an interesting question that we pose now.

Open Problem. Let $T$ be a nonexpansive mapping on $X$ which is asymptotically regular, and let $M=$ Fix $T$. Then, by Corollary 2.3, the algorithm converges:

$$
\lim _{n \rightarrow \infty}\left\|T^{n} x-P_{M} x\right\|=0 \quad \text { for each } x \in X .
$$

Is it true that the accelerated algorithm for $T$ also converges? That is, does the following hold:

$$
\lim _{n \rightarrow \infty}\left\|A_{T}^{n}(T x)-P_{M} x\right\|=0 \quad \text { for each } x \in X ?
$$

We have seen that the answer is affirmative in several special cases. For example, when any one of the following conditions are satisfied, then (3.24.9) holds.

(1) $T$ is selfadjoint and nonnegative (Theorem 3.20); in particular, if $T=$ $\left(P_{M_{k}} P_{M_{k-1}} \cdots P_{M_{1}}\right)^{*}\left(P_{M_{k}} P_{M_{k-1}} \cdots P_{M_{1}}\right)$ (Corollary 3.21 ).

(2) $T=P_{M_{2}} P_{M_{1}}$ is the product of two orthogonal projections (Theorem 3.23).

(3) $c(T)<1$ (Theorem 3.16); in particular, if $T=P_{M_{k}} P_{M_{k-1}} \cdots P_{M_{1}}$ and $M_{1}^{\perp}+M_{2}^{\perp}+\cdots+M_{k}^{\perp}$ is closed, then $c(T)<1$ (see [2]).

In particular, does (3.24.9) hold if $T$ is the product of $k \geq 3$ orthogonal projections? In this case, we can show that

$$
A_{T}^{n}(T x) \rightarrow P_{M} x \quad \text { weakly for each } x \in X .
$$

But we are not sure whether the convergence must be in norm.

To prepare for the last main result, we begin with a useful lemma.

Lemma 3.25. Define the function

$$
E(\alpha, \beta):=\frac{\beta-\alpha}{2-\alpha-\beta} \quad \text { for all } \alpha, \beta \in \mathbb{R} \text { with } \alpha+\beta \neq 2 .
$$

(1) Then $E$ is a continuously differentiable function on its domain such that

(a) $\frac{\partial E(\alpha, \beta)}{\partial \alpha}=\frac{2(\beta-1)}{(2-\alpha-\beta)^{2}}$, and

(b) $\frac{\partial E(\alpha, \beta)}{\partial \beta}=\frac{2(1-\alpha)}{(2-\alpha-\beta)^{2}}$.

In particular, if $c \leq 1$, then $E(\alpha, c)$ (respectively, $E(c, \beta)$ ) is a decreasing (respectively, increasing) function of $\alpha$ (respectively, $\beta$ ) in each of the two components of its domain.

(2) (a) $|E(\alpha, \beta)|<1$ if and only if $(1-\alpha)(1-\beta)>0$.

(b) $|E(\alpha, \beta)|=1$ if and only if $(1-\alpha)(1-\beta)=0$.

(c) $|E(\alpha, \beta)|>1$ if and only if $(1-\alpha)(1-\beta)<0$. 
Proof. The verification of (1) is easy.

(2) Write

$$
E(\alpha, \beta)=\frac{\beta-\alpha}{2-\alpha-\beta}=\frac{(1-\alpha)-(1-\beta)}{(1-\alpha)+(1-\beta)}=\frac{r_{1}-r_{2}}{r_{1}+r_{2}},
$$

where $r_{1}=1-\alpha$, and $r_{2}=1-\beta$. Clearly, $|E(\alpha, \beta)|<1$ if and only if $\left|\frac{r_{1}-r_{2}}{r_{1}+r_{2}}\right|<1$ if and only if $\left|r_{1}-r_{2}\right|<\left|r_{1}+r_{2}\right|$ if and only if $r_{1} r_{2}>0$. This proves (a). Also, $|E(\alpha, \beta)|=1$ if and only if $r_{1} r_{2}=0$, which proves (b). Finally, $|E(\alpha, \beta)|>1$ if and only if $\left|\frac{r_{1}-r_{2}}{r_{1}+r_{2}}\right|>1$ if and only if $r_{1} r_{2}<0$, which proves (c).

Lemma 3.26. Let $T$ be selfadjoint,

$$
c_{1}:=\inf \left\{\langle T x, x\rangle \mid x \in M^{\perp},\|x\|=1\right\},
$$

and

$$
c_{2}:=\sup \left\{\langle T x, x\rangle \mid x \in M^{\perp},\|x\|=1\right\},
$$

where both $c_{1}$ and $c_{2}$ are defined to be 0 if $M^{\perp}=\{0\}$, i.e., if $M=X$. Then

$$
\max \left\{c_{2},-c_{1}\right\}=c(T):=\left\|T P_{M^{\perp}}\right\| .
$$

Moreover, if $T$ is also nonnegative, then

$$
c_{2}=c(T)
$$

Proof. First note that

$$
-c_{1}=-\inf \left\{\langle T x, x\rangle \mid x \in M^{\perp},\|x\|=1\right\}=\sup \left\{-\langle T x, x\rangle \mid x \in M^{\perp},\|x\|=1\right\} .
$$

Hence,

$$
\begin{aligned}
\max \left\{c_{2},-c_{1}\right\}= & \sup \left\{|\langle T x, x\rangle| \mid x \in M^{\perp},\|x\|=1\right\} \\
= & \sup \left\{\left|\left\langle T P_{M^{\perp}} x, P_{M^{\perp}} x\right\rangle\right| \mid x \in X,\|x\|=1\right\} \\
= & \sup \left\{\left|\left\langle P_{M^{\perp}} T P_{M^{\perp}} x, x\right\rangle\right| \mid x \in X,\|x\|=1\right\} \\
= & \sup \left\{\left|\left\langle T P_{M^{\perp}} x, x\right\rangle\right| \mid x \in X,\|x\|=1\right\} \\
& \left(\text { using Lemma } 3.12 \text { and the idempotency of } P_{M^{\perp}}\right) \\
= & \left\|T P_{M^{\perp}}\right\| \\
& \left(\text { since } T P_{M^{\perp}}\right. \text { is selfadjoint and using [3, Proposition 2.13, p. 34]) } \\
= & c(T),
\end{aligned}
$$

which proves (3.26.3). Finally, if $T$ is also nonnegative, then $0 \leq c_{1} \leq c_{2}$ and so $\max \left\{c_{2},-c_{1}\right\}=c_{2}$. Thus (3.26.4) follows from (3.26.3).

Lemma 3.27. Let $T$ be selfadjoint and nonexpansive, and let $c_{1}$ and $c_{2}$ be defined as in (3.26.1) and (3.26.2). Then

$$
\left\|A_{T}(y)\right\| \leq\left(\frac{c_{2}-c_{1}}{2-c_{1}-c_{2}}\right)\|y\| \quad \text { for every } y \in M^{\perp} .
$$

In particular,

$$
\left\|A_{T}^{n}(y)\right\| \leq\left(\frac{c_{2}-c_{1}}{2-c_{1}-c_{2}}\right)^{n}\|y\| \quad \text { for every } y \in M^{\perp}, n \in \mathbb{N} .
$$


The inequality (3.27.2) follows from (3.27.1) by induction, using the fact that $A_{T}(y) \in M^{\perp}$ whenever $y \in M^{\perp}$ (Lemma 3.3(4)). Our proof of (3.27.1), just like that of Theorem 3.20, uses the spectral theorem. Before proving this lemma, let us state a few consequences of it.

Theorem 3.28. Let $T$ be selfadjoint and nonexpansive, and let $c_{1}$ and $c_{2}$ be defined as in (3.26.1) and (3.26.2). Then

$$
\left\|A_{T}^{n-1}(T x)-P_{M} x\right\| \leq\left(\frac{c_{2}-c_{1}}{2-c_{1}-c_{2}}\right)^{n-1} c(T)\left\|x-P_{M} x\right\|
$$

for every $x \in X$ and $n \in \mathbb{N}$.

Proof. Let $x \in X$ and set $y=T x-P_{M} x$. Then $y \in M^{\perp}$ by Lemma 3.3(6). Substitute this $y$ into $(3.27 .2)$ (and replace $n$ by $n-1$ ) to obtain

$$
\left\|A_{T}^{n-1}\left(T x-P_{M} x\right)\right\| \leq\left(\frac{c_{2}-c_{1}}{2-c_{1}-c_{2}}\right)^{n-1}\left\|T x-P_{M} x\right\| .
$$

But $A_{T}^{n-1}\left(T x-P_{M} x\right)=A_{T}^{n-1}(T x)-P_{M} x$ by Lemma 3.8(3) and

$$
\left\|T x-P_{M} x\right\|=\left\|T\left(x-P_{M} x\right)\right\| \leq c_{1}(T)\left\|x-P_{M} x\right\| \quad \text { by }(3.14 .1) .
$$

This proves (3.28.1).

Theorem 3.29. Let $T$ be selfadjoint, nonnegative, and nonexpansive. Then

$$
\left\|A_{T}^{n-1}(T x)-P_{M} x\right\| \leq \frac{c(T)^{n}}{[2-c(T)]^{n-1}}\left\|x-P_{M} x\right\| \quad \text { for every } x \in X, n \in \mathbb{N} \text {. }
$$

Proof. Since $T$ is nonnegative, $c_{1} \geq 0$ and $c(T)=c_{2}$ by Lemma 3.26. Since $T$ is nonexpansive, $c_{2} \leq 1$. Thus

$$
0 \leq c_{1} \leq c_{2}=c(T) \leq 1
$$

Then, using Theorem 3.28, we obtain that for every $x \in X$,

$$
\begin{aligned}
\left\|A_{T}^{n-1}(T x)-P_{M} x\right\| & \leq\left(\frac{c_{2}-c_{1}}{2-c_{1}-c_{2}}\right)^{n-1} c(T)\left\|x-P_{M} x\right\| \\
& =\left(\frac{c(T)-c_{1}}{2-c_{1}-c(T)}\right)^{n-1} c(T)\left\|x-P_{M} x\right\| .
\end{aligned}
$$

Now $\frac{c(T)-c_{1}}{2-c_{1}-c(T)}=E\left(c_{1}, c(T)\right), c_{1}$ and 0 are in the same component of the domain of $E(\cdot, c(T))$, and $E(\cdot, c(T))$ is a decreasing function by Lemma 3.25. This implies that

$$
\frac{c(T)-c_{1}}{2-c_{1}-c(T)}=E\left(c_{1}, c(T)\right) \leq E(0, c(T))=\frac{c(T)}{2-c(T)} .
$$

This together with (3.29.2) yields (3.29.1).

Remarks. Comparing (3.29.1) with (3.16.2), we see that for each selfadjoint, nonnegative, and nonexpansive operator $T$, it follows that

$$
\left\|A_{T}^{n-1}(T x)-P_{M} x\right\| \leq\left[\prod_{1}^{n-1} f\left(x_{i}\right)\right] c(T)^{n}\left\|x-P_{M} x\right\|
$$


and

$$
\left\|A_{T}^{n-1}(T x)-P_{M} x\right\| \leq \frac{c(T)^{n}}{[2-c(T)]^{n-1}}\left\|x-P_{M} x\right\| .
$$

Thus it is natural to ask whether one of these bounds is always better than the other. In other words, do either one of the following two inequalities always hold:

$$
\prod_{1}^{n-1} f\left(x_{i}\right) \leq \frac{1}{[2-c(T)]^{n-1}} \quad \text { for all } n \geq 2
$$

or

$$
\frac{1}{[2-c(T)]^{n-1}} \leq \prod_{1}^{n-1} f\left(x_{i}\right) \quad \text { for all } n \geq 2 ?
$$

We now show that neither of these two inequalities always holds. To see that inequality (b) does not always hold, consider the example when $X=\ell_{2}(2)$ is the Euclidean plane, $M_{1}$ (resp., $M_{2}$ ) is the horizontal (resp., vertical) axis, and $T=P_{M_{1}} P_{M_{2}} P_{M_{1}}$. Then $T=0, M=$ Fix $T=\{0\}, c(T)=\left\|T P_{M^{\perp}}\right\|=0, f(x)=0$ for all $x \in \ell_{2}(2)$, and $\frac{1}{2-c(T)}=\frac{1}{2}$. Hence

$$
\prod_{1}^{n} f\left(x_{i}\right)<\frac{1}{[2-c(T)]^{n}} \quad \text { for every } n \geq 1 .
$$

To see that (a) does not always hold, let $X=\ell_{2}(2)$ denote the Euclidean plane and define $T$ on $X$ by $T\left(\alpha e_{1}+\beta e_{2}\right)=\frac{99}{100} \alpha e_{1}+\frac{19}{100} \beta e_{2}$. Then $T$ is a nonnegative selfadjoint linear operator on $X, M=\operatorname{Fix} T=\{0\}$, and $c(T)=\|T\|=\frac{99}{100}$. Letting $x_{0}:=\frac{10}{11} e_{1}+\frac{10}{19} e_{2}$, we can easily deduce that $x_{1}:=T x_{0}=\frac{9}{10} e_{1}+\frac{1}{10} e_{2}, T x_{1}=$ $\frac{891}{1000} e_{1}+\frac{19}{1000} e_{2}, t_{x_{1}}=\frac{\left\langle x_{1}, x_{1}-T x_{1}\right\rangle}{\left\|x_{1}-T x_{1}\right\|^{2}}=\frac{100}{41}$, and $A_{T}\left(x_{1}\right)=t_{x_{1}} T x_{1}+\left(1-t_{x_{1}}\right) x_{1}=$ $\frac{36}{41} e_{1}-\frac{4}{41} e_{2}$. Hence, $f\left(x_{1}\right)=\frac{\left\|A_{T}\left(x_{1}\right)\right\|}{\left\|T x_{1}\right\|}=\frac{1000}{41}\left(\frac{656}{397121}\right)^{\frac{1}{2}}=0.9913034925 \cdots$ and $\frac{1}{2-c(T)}=\frac{100}{101}=0.9900990099 \cdots$ implies that

$$
\frac{1}{2-c(T)}<f\left(x_{1}\right)
$$

so (a) fails for $n=2$.

Proof of Lemma 3.27. We should first note that $c_{1}+c_{2}<2$, and hence the expressions on the right side of both (3.27.1) and (3.27.2) are well-defined. For otherwise, $c_{1}=c_{2}=1$ and $\langle x, T x\rangle=1$ for all $x \in M^{\perp}$ with $\|x\|=1$. By the condition of equality in the Schwarz inequality, this implies that $x=T x$ for all $x \in M^{\perp}$. That is, $M^{\perp} \subset M$ and so $M^{\perp}=\{0\}$. But this implies that $c_{1}=c_{2}=0$, a contradiction. It follows also that $E\left(c_{1}, c_{2}\right) \geq 0$.

In the notation of Lemma 3.25, we must show that

$$
\left\|A_{T}(y)\right\| \leq E\left(c_{1}, c_{2}\right)\|y\| \quad \text { for every } y \in M^{\perp} .
$$

If $M^{\perp}=\{0\}$, then (3.27.2) is obvious; both sides are in fact 0 . Thus we can assume $M^{\perp} \neq\{0\}$. Fix any $y \in M^{\perp} \backslash\{0\}$. By scaling and Lemma 3.8(4), we may assume $\|y\|=1$. Let

$$
N:=\operatorname{span}\{y, T y\}
$$


Then $N \subset M^{\perp}$ by Lemma 3.3(4) and $1 \leq \operatorname{dim} N \leq 2$. If $\operatorname{dim} N=1$, then $T y=\alpha y$ for some scalar $\alpha \neq 1$ and thus

$$
0 \in \operatorname{span}\{y\}=\operatorname{span}\{y, T y\}=\operatorname{aff}\{y, T y\}
$$

implies $A_{T}(y)=0$ since $A_{T}(y)$ is the point in aff $\{y, T y\}$ having minimal norm by Theorem 3.7. Hence, (3.27.2) holds and we may therefore assume that $\operatorname{dim} N=2$. In particular, $T y \notin \operatorname{span}\{y\}$.

Define the operator $S:=P_{N} T P_{N}$. Then $S$ is a compact selfadjoint (nonexpansive) operator with $\mathcal{R}(S) \subset N$, and thus $n:=\operatorname{dim} \mathcal{R}(S) \leq 2$. But both $y$ and $T y$ are in $N$; so

$$
S y=P_{N} T P_{N} y=P_{N} T y=T y
$$

implies that $T y \in \mathcal{R}(S)$ and hence $1 \leq n \leq 2$. By the spectral theorem 3 . Corollary 5.4 , p. 47], there exist an orthornormal basis $\left\{e_{i}\right\}_{1}^{n}$ of $\mathcal{N}(S)^{\perp}(=\mathcal{R}(S))$ and scalars $\left\{\lambda_{i}\right\}_{1}^{n}$ such that

$$
S x=\sum_{1}^{n} \lambda_{i}\left\langle x, e_{i}\right\rangle e_{i} \quad \text { for every } x \in X .
$$

In particular,

$$
S e_{j}=\lambda_{j} e_{j} \quad(j=1, \ldots, n)
$$

so each $e_{j}$ is an eigenvector of $S$ with eigenvalue $\lambda_{j}$. Also,

$$
\begin{aligned}
\lambda_{j} & =\left\langle\lambda_{j} e_{j}, e_{j}\right\rangle=\left\langle S e_{j}, e_{j}\right\rangle=\left\langle P_{N} T P_{N} e_{j}, e_{j}\right\rangle \\
& =\left\langle T P_{N} e_{j}, P_{N} e_{j}\right\rangle=\left\langle T e_{j}, e_{j}\right\rangle
\end{aligned}
$$

since each $e_{j} \in \mathcal{R}(S) \subset N$. Since $N \subset M^{\perp}$, this proves that

$$
c_{1} \leq \lambda_{j} \leq c_{2} \quad(j=1, \ldots, n) .
$$

We consider two cases.

Case 1. $n=1$.

Then since

$$
N=\mathcal{R}(S) \oplus\left[\mathcal{R}(S)^{\perp} \cap N\right],
$$

$\operatorname{dim} N=2$, and $\operatorname{dim} \mathcal{R}(S)=1$, it follows that $\operatorname{dim}\left[\mathcal{R}(S)^{\perp} \cap N\right]=1$. Hence we can choose $e_{2} \in \mathcal{R}(S)^{\perp} \cap N$ with $\left\|e_{2}\right\|=1$ and define $\lambda_{2}=0$. Then $\left\{e_{1}, e_{2}\right\}$ is a basis for $N$, and $S e_{2}=0=\lambda_{2} e_{2}$. If follows that (3.27.3)-(3.27.6) hold with $n=2$.

Case 2. $n=2$.

Then $\mathcal{R}(S)=N$ and (3.27.3)-(3.27.6) holds with $n=2$.

Thus each case can be reduced to the case when $n=2$.

If $E\left(c_{1}, c_{2}\right) \geq 1$, then (3.27.2) is obvious since then

$$
\left\|A_{T}(x)\right\| \leq\|x\| \leq E\left(c_{1}, c_{2}\right)\|x\|
$$

for each $x$, where (3.7.5) was used for the first inequality. Thus, we may assume that $0 \leq E\left(c_{1}, c_{2}\right)<1$. By Lemma 3.25, this is equivalent to $\left(1-c_{1}\right)\left(1-c_{2}\right)>0$. That is, either $1-c_{1}>0$ and $1-c_{2}>0$, or $1-c_{1}<0$ and $1-c_{2}<0$. But the latter inequality implies $c_{2}>1$ which contradicts the nonexpansiveness of $T$. Thus, we must have $1-c_{1}>0$ and $1-c_{2}>0$. That is,

$$
-1 \leq c_{1} \leq \lambda_{j} \leq c_{2}<1 \quad(j=1,2),
$$


where the lower bound $c_{1} \geq-1$ is also a consequence of the nonexpansiveness of $T$.

Moreover, since $\left\{e_{1}, e_{2}\right\}$ is an orthonormal basis for $N$ and since $y$ and $T y$ are in $N$, we have $y=\sum_{1}^{2} \alpha_{i} e_{i}$ and $T y=S y=\sum_{1}^{2} \lambda_{i} \alpha_{i} e_{i}$, where $\alpha_{i}:=\left\langle y, e_{i}\right\rangle(i=1,2)$. Then by (3.5.3) and using the fact that $\alpha_{1}^{2}+\alpha_{2}^{2}=\|y\|^{2}=1$, we deduce that

$$
\begin{aligned}
\left\|A_{T}(y)\right\|^{2} & =\|y\|^{2}-\frac{\langle y, y-T y\rangle^{2}}{\|y-T y\|^{2}} \\
& =1-\frac{\left\langle\sum_{1}^{2} \alpha_{i} e_{i}, \sum_{1}^{2} \alpha_{i} e_{i}-\sum_{1}^{2} \lambda_{i} \alpha_{i} e_{i}\right\rangle^{2}}{\left\|\sum_{1}^{2} \alpha_{i} e_{i}-\sum_{1}^{2} \lambda_{i} \alpha_{i} e_{i}\right\|^{2}} \\
& =1-\frac{\left[\sum_{1}^{2} \alpha_{i}^{2}\left(1-\lambda_{i}\right)\right]^{2}}{\sum_{1}^{2} \alpha_{i}^{2}\left(1-\lambda_{i}\right)^{2}} .
\end{aligned}
$$

Putting the expression on the right over a common denominator, expanding, and simplifying, we obtain

$$
\left\|A_{T}(y)\right\|^{2}=\frac{\alpha_{1}^{2} \alpha_{2}^{2}\left(\lambda_{2}-\lambda_{1}\right)^{2}}{\alpha_{1}^{2}\left(1-\lambda_{1}\right)^{2}+\alpha_{2}^{2}\left(1-\lambda_{2}\right)^{2}} .
$$

If $\lambda_{1}=\lambda_{2}$, then (3.27.8) implies that $A_{T}(y)=0$ and (3.27.2) is obvious. Thus we may assume that $\lambda_{1} \neq \lambda_{2}$. In fact, by reindexing if necessary, we may assume that $\lambda_{1}<\lambda_{2}$. Define $h:[0,1] \rightarrow \mathbb{R}$ by

$$
h(t):=\frac{t(1-t)\left(\lambda_{2}-\lambda_{1}\right)^{2}}{t\left(1-\lambda_{1}\right)^{2}+(1-t)\left(1-\lambda_{2}\right)^{2}} .
$$

Since $\alpha_{1}^{2}+\alpha_{2}^{2}=1$, we see that (3.27.8) implies that

$$
\left\|A_{T}(y)\right\|^{2} \leq \max \{h(t) \mid 0 \leq t \leq 1\} .
$$

But $h(0)=h(1)=0$ and $h(t)>0$ for all $0<t<1$. Hence the maximum of $h$ over $[0,1]$ occurs for some $t \in(0,1)$ that satisfies $h^{\prime}(t)=0$. Differentiating $h$ and expanding, we deduce that

$$
\left[t a^{2}+(1-t) b^{2}\right]^{2} h^{\prime}(t)=(a-b)^{2}[t(b-a)-b][t(a+b)-b],
$$

where $0<b:=1-\lambda_{2}<1-\lambda_{1}=: a$. Hence $h^{\prime}(t)=0$ if and only if $t=b /(b-a)<0$ or $t=b /(a+b) \in(0,1)$. Hence the maximum of $h$ over $[0,1]$ is attained at $t=b /(a+b)$. Thus

$$
\max _{0 \leq t \leq 1} h(t)=h\left(\frac{b}{a+b}\right)=\left(\frac{a-b}{a+b}\right)^{2}=\left(\frac{\lambda_{2}-\lambda_{1}}{2-\lambda_{2}-\lambda_{1}}\right)^{2}=E\left(\lambda_{1}, \lambda_{2}\right)^{2} .
$$

Combining this with (3.27.10), we obtain that $\left\|A_{T}(y)\right\|^{2} \leq E\left(\lambda_{1}, \lambda_{2}\right)^{2}$ or, equivalently,

$$
\left\|A_{T}(y)\right\| \leq\left|E\left(\lambda_{1}, \lambda_{2}\right)\right|=E\left(\lambda_{1}, \lambda_{2}\right) .
$$

By Lemma $3.25, E\left(\cdot, \lambda_{2}\right)$ is a decreasing function so that by $(3.27 .7)$, we get

$$
E\left(\lambda_{1}, \lambda_{2}\right) \leq E\left(c_{1}, \lambda_{2}\right) .
$$

On the other hand, by Lemma $3.25, E\left(c_{1}, \cdot\right)$ is an increasing function. By (3.27.7), it follows that

$$
E\left(c_{1}, \lambda_{2}\right) \leq E\left(c_{1}, c_{2}\right) .
$$


Combining (3.27.11)-(3.27.13), we obtain

$$
\left\|A_{T}(y)\right\| \leq E\left(c_{1}, c_{2}\right)
$$

and this is just (3.27.2).

Remarks. It is perhaps worth noting that the inequality (3.27.2), and hence the main inequality in each of Theorems 3.28 and 3.29, is sharp, at least for a large class of operators $T$. More precisely, one can prove the following result. If $T: X \rightarrow X$ is selfadjoint, nonexpansive, has finite rank, and is not the identity, then there exists $x^{*} \in M^{\perp}$ with $\left\|x^{*}\right\|=1$ and

$$
\left\|A_{T}^{n} x^{*}\right\|=\left(\frac{c_{2}-c_{1}}{2-c_{1}-c_{2}}\right)^{n}\left\|x^{*}\right\| \text { for } n=0,1,2, \ldots
$$

Our proof of this result was divided into two cases: when $\mathcal{R}(T) \neq X$ and when $\mathcal{R}(T)=X$. Since the proof was somewhat lengthy, we have omitted it.

Finally, we should mention that there are examples of expansive, selfadjoint, and positive mappings $T$ for which the algorithm (3.1.3) diverges for every nonzero $x$, but the accelerated counterpart (3.1.4) converges! That is, it is not always necessary to have the original algorithm converging to be able to accelerate it.

For example, let $X$ be the Euclidean plane $\ell_{2}(2)$ and define $T: X \rightarrow X$ by $T x=3 x(1) e_{1}+4 x(2) e_{2}$. Then $T$ is selfadjoint and positive, $M:=\operatorname{Fix} T=\{0\}$, and $\|T\|=4$ (so $T$ is expansive). However, $\left\|T^{n} x\right\| \geq 3^{n}\|x\|$ and $\left\|A_{T}^{n}(T x)\right\| \leq$ $3^{-n+1}\|x\|$ for every $x$. This shows that $\left\|T^{n} x-P_{M} x\right\| \rightarrow \infty$ for each $x \neq 0$, while $\left\|A_{T}^{n-1}(T x)-P_{M} x\right\| \rightarrow 0$ for each $x$.

Added in proof. Recently, there has been related work that has appeared since this paper was first submitted to the Transactions in July of 1999.

First, the authors of this paper showed that the iterates $x_{0}=x, x_{n}=A_{T}\left(T x_{n-1}\right)$ for $n \geq 1$ generated by the accelerated map for a linear nonexpansive map $T$ converge weakly to $P_{\mathrm{Fix} T}(x)$ (Fejér monotonicity and weak convergence of an accelerated method of projections, Canadian Math. Soc., Conference Proceedings, $\mathbf{2 7}(2002), 1-6)$. This generalizes the relation (3.24.10) above.

F. Deutsch (Accelerating the convergence of the method of alternating projections via a line search: a brief survey, in Inherently Parallel Algorithms in Feasibility and Optimization and their Applications (edited by D. Butnariu, Y. Censor, and S. Reich), 2001, Elsevier Science, 203-217) gave a survey of line search methods for accelerating the convergence of the method of alternating projections.

J. Xu and L. Zikatanov (The method of alternating projections and the method of subspace corrections in Hilbert space, J. Amer. Math. Soc., 15(2002), 573-597) gave an identity for estimating the norm of a product of nonexpansive linear operators on a Hilbert space.

\section{REFERENCES}

1. N. Aronszajn, Theory of reproducing kernels, Trans. Amer. Math. Soc., 68(1950), 337-404. MR 14: $479 \mathrm{c}$

2. H. H. Bauschke, J. M. Borwein, and A. S. Lewis, The method of cyclic projections for closed convex sets in Hilbert space, Recent developments in optimization theory and nonlinear analysis (Jerusalem, 1995), 1-38, Contemporary Mathematics 204, Amer. Math. Soc., Providence, R.I., 1997. MR 98c:49069

3. J. B. Conway, A Course in Functional Analysis (second edition), Graduate Texts in Mathematics 96, Springer-Verlag, New York, 1990. MR 91e:46001 
4. L. Debnath and P. Mikusinski, Introduction to Hilbert Spaces with Applications (second edition), Academic Press, San Diego, CA, 1999. MR 99k:46001

5. F. Deutsch, Rate of convergence of the method of alternating projections, Parametric Optimization and Approximation, ISNM 72 (B. Brosowski and F. Deutsch, eds.), Birkhäuser, Basel, 1985, pp. 96-107. MR 88d:41026

6. F. Deutsch, The method of alternating orthogonal projections, in Approximation Theory, Spline Functions and Applications (S. P. Singh, ed.), Kluwer Academic Publ., Dordrecht, 1992, pp. 105-121. MR 93a:41047

7. F. Deutsch, The angle between subspaces of a Hilbert space, in Approximation Theory, Wavelets and Applications (S.P. Singh, ed.), Kluwer Academic Publ., Dordrecht, pp. 107130. MR 96e: 46027

8. F. Deutsch and H. Hundal, The rate of convergence of Dykstra's cyclic projections algorithm: the polyhedral case, Numer. Funct. Anal. and Optimiz. 15 no. 5-6 (1994), 537-565. MR 95f:49047

9. F. Deutsch and H. Hundal, The rate of convergence for the method of alternating projections, II, J. Math. Anal. Appl. 205 (1997), 381-405. MR 97i:41025

10. J. Dyer, Acceleration of the convergence of the Kaczmarz method and iterated homogeneous transformations, doctoral dissertation (1965).

11. C. Franchetti and W. Light, On the von Neumann alternating algorithm in Hilbert space, J. Math. Anal. Appl. 114 (1986), 305-314. MR 87f:41058

12. K. Friedrichs, On certain inequalities and characteristic value problems for analytic functions and functions of two variables, Trans. Amer. Math. Soc. 41 (1937), 321-364.

13. W. B. Gearhart and M. Koshy, Acceleration schemes for the method of alternating projections, J. Comp. Appl. Math. 26 (1989), 235-249. MR 90h:65095

14. L. G. Gubin, B. T. Polyak, and E. V. Raik, The method of projections for finding the common point of convex sets, USSR Computational Mathematics and Mathematical Physics $7(6)$ (1967), 1-24.

15. I. Halperin, The product of projection operators, Acta. Sci. Math. (Szeged) 23 (1962), 96-99. MR 25:5373

16. M. Hanke and W. Niethammer, On the acceleration of Kaczmarz's method for inconsistent linear systems, Linear Algebra Appl. 130 (1990), 83-98. MR 91f:65065

17. S. Kayalar and H. Weinert, Error bounds for the method of alternating projections, Math. Control Signals Systems 1 (1988), 43-59. MR 89b:65137

18. J. von Neumann, Functional Operators. II, Princeton University Press, Princeton, NJ, 1950. [This is a reprint of mimeograghed lecture notes first distributed in 1933.] MR 11:599e

19. F. Riesz and B. Sz.-Nagy, Über Kontraktionen des Hilbertschen Raumes, Acta. Sci. Math. 10 (1941-1943), 202-205. MR 8:35a

20. F. Riesz and B. Sz.-Nagy, Functional Analysis, Ungar, New York, 1955. MR 17:175i

21. R. Smarzewski, Iterative recovering of orthogonal projections, preprint (December, 1996).

22. K. T. Smith, D. C. Solmon, and S. L. Wagner, Practical and mathematical aspects of the problem of reconstructing objects from radiographs, Bull. Amer. Math. Soc. 83 (1976), 12271270. MR 58:9394a

Department of Mathematics and Statistics, Okanagan University College, Kelowna, British Columbia, Canada V1V 1 V7

E-mail address: bauschke@cecm.sfu.ca

Current address: Department of Mathematics and Statistics, University of Guelph, Guelph, Ontario, Canada N1G 2W1

Department of Mathematics, The Pennsylvania State University, University Park, Pennsylvania 16802

E-mail address: deutsch@math.psu.edu

NONRAND, 12100 Wiltshire \#1650, Los Angeles, California 90025

E-mail address: hundalhm@vicon.net

Current address: 146 Cedar Ridge Drive, Port Matilda, Pennsylvania 16870

Department of Mathematics, Sogang University, Seoul, Korea

E-mail address: shpark@ccs.sogang.ac.kr 